\title{
Molecular Dynamics Simulations of Alzheimer's $\beta$-Amyloid Protofilaments
}

\author{
Nicolae-Viorel Buchete, Robert Tycko and Gerhard Hummer*
}

Laboratory of Chemical Physics National Institute of Diabetes and Digestive and Kidney Diseases, National Institutes of Health, Bethesda, MD 20892-0520, USA
Filamentous amyloid aggregates are central to the pathology of Alzheimer's disease. We use all-atom molecular dynamics (MD) simulations with explicit solvent and multiple force fields to probe the structural stability and the conformational dynamics of several models of Alzheimer's $\beta$-amyloid fibril structures, for both wild-type and mutated amino acid sequences. The structural models are based on recent solid state NMR data. In these models, the peptides form in-register parallel $\beta$-sheets along the fibril axis, with dimers of two U-shaped peptides located in layers normal to the fibril axis. Four different topologies are explored for stacking the $\beta$-strand regions against each other to form a hydrophobic core. Our MD results suggest that all four NMR-based models are structurally stable, and we find good agreement with dihedral angles estimated from solid-state NMR experiments. Asp23 and Lys28 form buried salt-bridges, resulting in an alternating arrangement of the negatively and positively charged residues along the fibril axis that is reminiscent of a one-dimensional ionic crystal. Interior water molecules are solvating the buried salt-bridges. Based on data from NMR measurements and MD simulations of short amyloid fibrils, we constructed structural models of long fibrils. Calculated X-ray fiber diffraction patterns show the characteristics of packed $\beta$-sheets seen in experiments, and suggest new experiments that could discriminate between various fibril topologies.

Published by Elsevier Ltd.

Keywords: amyloid fibrils; molecular dynamics; Alzheimer's disease; fibril structure; molecular hydration design and development of new types of nanomaterials. $^{7-9}$ However, high-resolution structures are not currently available for $A \beta$ fibrils because of the inherent non-crystallinity and insolubility of these materials. Until approximately ten years ago, information about the molecular structures of amyloid fibrils was limited to the presence of ribbon-like $\beta$-sheets arranged in a cross- $\beta$ orientation, as indicated by the $4.7-4.8 \AA$ meridional reflection in $\mathrm{X}$-ray fiber diffraction data. ${ }^{1}$

Recently, significant progress has been made toward the elucidation of the molecular structures of amyloid fibrils. ${ }^{4,5}$ With solid state nuclear magnetic resonance (NMR) methods, specific interatomic distances that constrain the supramolecular organization can be measured, and dihedral angles and distances that constrain the molecular conformation can be estimated. Solid state NMR data and the resulting structural models ${ }^{10-14}$ support a parallel $\beta$-sheet structure in fibrils formed by both the 40 and 42-residue $\beta$-amyloid peptides $\left(\mathrm{A} \beta_{1-40,42}\right)$. Parallel $\beta$-sheets 
have been found by solid state NMR originally in fibrils formed by residues 10-35 of $\beta$-amyloid $\left(\mathrm{A} \beta_{10-35}\right) .{ }^{12,15,16}$ However, antiparallel $\beta$-sheets have also been found by solid state NMR in fibrils formed by shorter $\beta$-amyloid fragments. ${ }^{17-20}$ It is important to note that recent solid state NMR and electron microscopy experiments have shown that $\mathrm{A} \beta_{1-40}$ can form at least two distinct amyloid fibril structures, with distinct and self-propagating morphologies and molecular-level structural features, dependent on subtle variations in fibril growth conditions. ${ }^{4}$ Although all $\mathrm{A} \beta_{1-40}$ fibril structures studied to date contain parallel $\beta$-sheets, distinct structures differ in the specific details of side-chain-side-chain contacts and in mass-perlength (MPL) values. All results discussed below are based on experimental data for fibrils grown with gentle agitation, under conditions described by Petkova et al. ${ }^{4}$

The amino acid sequence of the $A \beta_{1-42}$ peptide is $\mathrm{D}_{1} \mathrm{AEFRHDSG}_{9}$ YEVHHQKLVFFAED $_{23}$ VGSNK $_{28}$ GAIIGLMVGGVV ${ }_{40} \mathrm{IA}_{42}$, where subscripts indicate residue numbers. The solid state NMR data are consistent with an $A \beta_{1-40}$ monomer secondary structure composed of a structurally disordered $N$-terminal region followed by two $\beta$-strand segments connected by a "loop" or "bend" segment. $^{10,21}$ Other recent experimental ${ }^{22-25}$ and statistical studies ${ }^{26}$ also support models with a $\beta$-sheet core and a single loop near residues 23-26. Scanning transmission electron microscopy (STEM) data $4,10,12,27$ indicate that $A \beta_{1-40}$ and $A \beta_{1-42}$ fibrils with minimal MPL (which we call protofilaments, see Methods) contain two peptide molecules per layer in $\beta$-sheet motifs.

The objective here is to use molecular dynamics (MD) simulations to add further structural detail to the solid state NMR models for $A \beta_{1-40}$ fibrils, and to explore the structural stability, hydration and dynamics of peptide fibrils. Several MD simulations of $\beta$-amyloid peptide fragments have been reported, ${ }^{28-37}$ providing insights into the molecular properties of monomers and small oligomers of fibril-forming peptides. Ma \& Nussinov ${ }^{30}$ performed an extensive MD analysis of various structural models of short $\beta$-amyloid fragments, including parallel and antiparallel $\beta$-sheet structures. In agreement with the NMR experiments, their simulations suggested a strand-loop-strand structure with parallel $\beta$-sheets for $A \beta_{10-35}$ fibrils. Moreover, Ma \& Nussinov also concluded from their simulations that an interior salt-bridge between residues D23 and K28 formed a major element of their fibril structures, consistent with the NMR data. ${ }^{30}$ Preceding the solid state NMR measurements demonstrating parallel $\beta$-sheets, atomic models of antiparallel pleated $\beta$-sheet structures of $A \beta$ protofilaments ${ }^{38-41}$ were also explored using MD simulations. ${ }^{42-44}$

Here, we perform a computational study employing all-atom MD simulations starting from the NMR-derived structural models of Alzheimer's $\beta$-amyloid fibrils. In light of experimental evidence that the $\mathrm{N}$-terminal residues $1-8$ are disordered, ${ }^{10,25,45,46}$ we use the $A \beta_{9-40}$ segment as a model for the full-length amyloid peptides. The structurally detailed solid state NMR models ${ }^{5,10}$ provide a set of realistic starting configurations for our all-atom simulations of $\mathrm{A} \beta$ protofilaments. Explicit-solvent MD simulations of molecular systems with 17,000-52,000 atoms are used to explore possible fibril topologies.

After a brief description of the different fibril topologies and the MD simulations of amyloid peptides in finite fibril segments and infinitely long fibrils, we explore the conformational stability of various fibril topologies. We then characterize in detail the structural characteristics of simulated amyloid fibrils, such as their backbone dihedral angles, the left-handed twisted configuration of the protofilaments, their molecular surface, interior hydration and internal salt-bridges. Based on fibril symmetry transformations extracted from MD simulations of short fibril segments, we build models of long amyloid fibrils. Finally, we compare structural aspects of the models to experiment, in particular the MPL and twisting characteristics of long amyloid fibrils, and the calculated X-ray fiber diffraction patterns. Details about simulation and analysis procedures are described in Methods.

\section{Results and Discussion}

\section{Atomic-level structure of $A \beta$ protofilaments}

In this work, we study atomic-level models of structures of molecular fibrils composed of parallel dimeric layers of $A \beta_{9-40}$ wild-type peptides with the sequence $\mathrm{G}_{9} \mathrm{YEVHHQKLVFFAED}_{23} \mathrm{VGSNK}_{28^{-}}$ GAIIGLMVGGVV 40 . As indicated by solid state NMR measurements, ${ }^{4,10,21}$ residues V12-V24 and A30-V40 adopt $\beta$-strand backbone conformations, residues G25-G29 are part of a non- $\beta$ structure, and residues D23 and K28 form a salt-bridge. The two $\beta$-strand regions form in-register parallel $\beta$-sheets with the corresponding residues from the neighboring $A \beta_{9-40}$ molecules in the fibril. ${ }^{4,13,14}$ The minimal experimentally detected structural unit (i.e., the protofilament) contains two strands of $A \beta_{9-40}$ molecules, as suggested by the STEM data. $4,10,12,27$ Based on this experimental information, we generate several atomically detailed models of $\mathrm{A} \beta_{9-40}$ fibrils as described next, and we investigate them using MD simulations. The all-atom, NPT (NPT assumes a fixed number of particles, $N$, constant pressure, $P$, and temperature, $T$ ) simulations in explicit solvent performed in this work are summarized in Table 1, with a detailed description given in Methods.

Initially, the fibrils are aligned with their hydrogen bonding direction (or growth axis) along the $z$-axis. Planes normal to the $z$-axis contain dimeric layers of $A \beta_{9-40}$, so that the initial MPL value for $A \beta_{9-40}$ is approximately $18 \mathrm{kDa} / \mathrm{nm}$ (two peptides of $4.3 \mathrm{kDa}$ molecular mass per layer, with a 
Table 1. Summary of the molecular dynamics simulations of amyloid protofilaments

\begin{tabular}{|c|c|c|c|c|}
\hline Notation & $N_{\text {total }}$ & $N_{\text {wat }}$ & Time (ns) & Force field \\
\hline \multicolumn{5}{|c|}{ Finite fibril sustem } \\
\hline$C_{2}^{x}$ & 47,498 & 14,566 & 10.0 & CHARMM \\
\hline$C_{2}^{z}$ & 51,695 & 15,965 & 10.0 & CHARMM \\
\hline$F C_{2}^{x}$ & 50,588 & 15,596 & 10.0 & CHARMM \\
\hline$F C_{2}^{z}$ & 52,250 & 16,150 & 10.0 & CHARMM \\
\hline$F C_{2}^{x}-A$ & 32,915 & 9697 & 10.0 & AMBER \\
\hline$F C_{2}^{x}-A G S$ & 32,915 & 9697 & 10.0 & AMBER-GS \\
\hline \multicolumn{5}{|c|}{ Periodic infinite fibril system } \\
\hline$C_{2}^{x}$ & 23,918 & 6706 & 10.0 & CHARMM \\
\hline$M C_{2}^{x}$ & 17,575 & 4629 & 6.0 & CHARMM \\
\hline$C_{2}^{z}$ & 21,083 & 5761 & 10.0 & CHARMM \\
\hline$F C_{2}^{x}$ & 24,230 & 6810 & 10.0 & CHARMM \\
\hline$F C_{2}^{z}$ & 25,013 & 7071 & 10.0 & CHARMM \\
\hline \multicolumn{5}{|c|}{ Finite fibril system continuing an infinite system simulation } \\
\hline$F C_{2}^{x}$ & 32,693 & 9631 & 10.0 & CHARMM \\
\hline
\end{tabular}

Most simulations were performed using the CHARMM27 force field.$^{73}$ For the simulations denoted by $F C_{2}^{x}-A$ and for $F C_{2}^{x}$ - $A G S$, we used the AMBER94 force field, ${ }^{74}$ and a modified AMBER94 force field, ${ }^{75}$ respectively. $N_{\text {total }}$ and $N_{\text {wat }}$ are the numbers of atoms and the number of water molecules in the simulation system, respectively.

separation of $4.8 \AA$ along the fibril axis). The $x$-axis is taken to be parallel with the two $\beta$-strand regions V12-V24 and A30-V40. Following this convention, we denote the various fibril configurations according to their symmetry (Figure 1 ). In the $C_{2}^{x}$ topology, the two peptides in the dimeric layers are related through a $180^{\circ}$ rotation about the $x$-axis
(Figure 1(a)). In the $C_{2}^{z}$ fibril topology, the two $A \beta$ peptides are related by a $180^{\circ}$ rotation about the $z$-axis (Figure 1(b)). We also consider topologies in which the backbone of the C-terminal $\beta$-strand is rotated by $180^{\circ}$ about its axis, thereby exchanging the intramolecular and intermolecular hydrophobic contacts of side-chains in the C-terminal $\beta$-strand. (a)

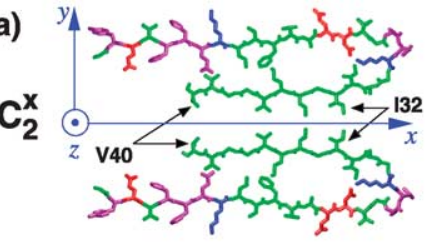

(b)

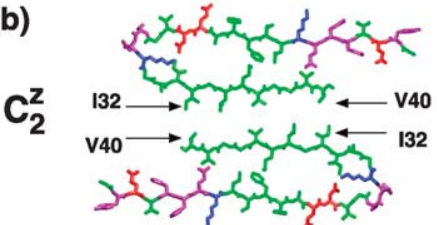

(c)

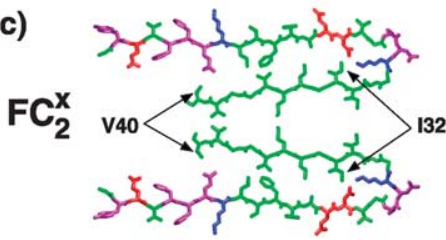

(d)

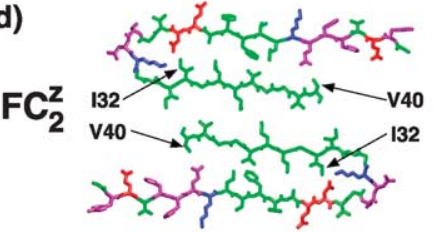

$0.0 \mathrm{~ns}$
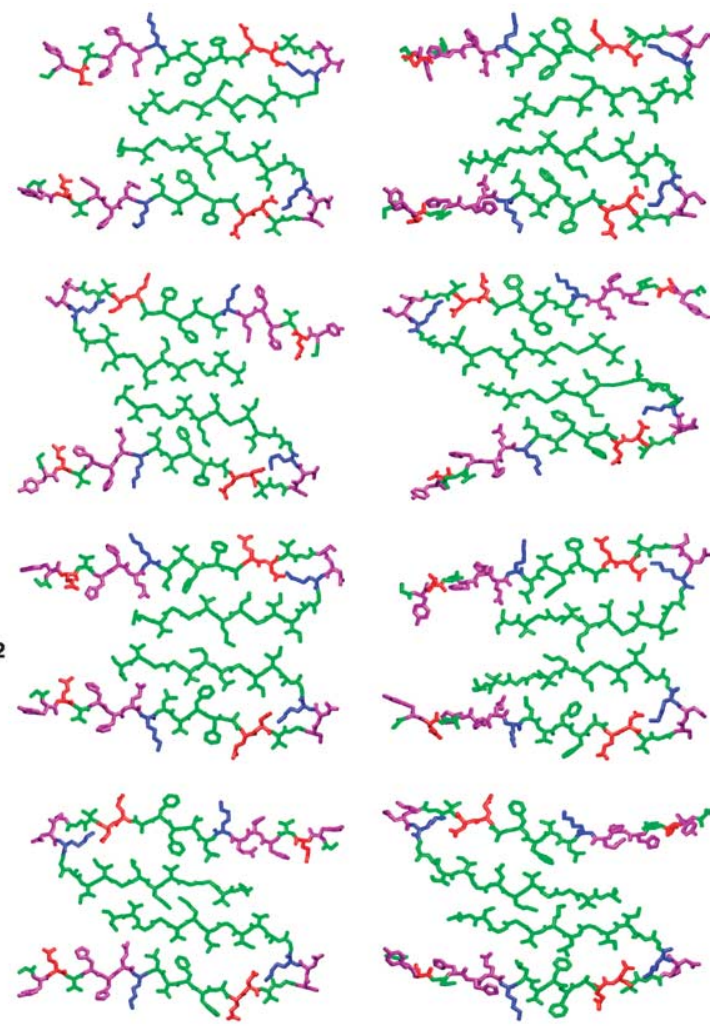

$0.1 \mathrm{~ns}$

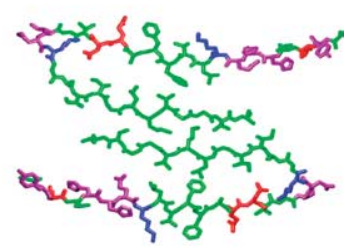

$10.0 \mathrm{~ns}$

Figure 1. Structures of dimeric layers of the $\mathrm{A} \beta_{9-40}$ system during the MD simulations. Shown are structures of models with (a) $C_{2}^{x}$, (b) $C_{2}^{z}$, (c) $F C_{2}^{x}$, and (d) $F C_{2}^{z}$ symmetry at the beginning of the simulations (left), after $0.1 \mathrm{~ns}$ (center), and at the end of the simulations (right, $10 \mathrm{~ns}$ ). The colors correspond to the amino acid types (green for hydrophobic, magenta for polar, red for acidic and blue for basic). 
These "flipped" topologies $\left(F C_{2}^{x}\right.$ and $\left.F C_{2}^{z}\right)$ are generated by rotating the backbone $\Phi$ and $\Psi$ dihedral angles in the vicinity of residues G29 and A30. Such flipped topologies were considered in the simulations reported by $\mathrm{Ma} \&$ Nussinov. ${ }^{30}$ In the $F C_{2}^{x}$ and $F C_{2}^{z}$ topologies, the residues forming intermolecular side-chain interactions in the dimeric layers are I31-G33-M35-G37-V39 (i.e., instead of I32-L34-V36-G38-V40 in $C_{2}^{x}$ and $C_{2}^{z}$ topologies).

For each of the four topologies $\left(C_{2}^{x}, C_{2}^{z}, F C_{2}^{x}\right.$, and $F C_{2}^{z}$ ), atomically detailed protofilament structures are built starting with energy-minimized atomic co-ordinates for the structural model proposed by Petkova et al. ${ }^{10}$ In these MD simulations, we study (a) finite fibrils consisting of eight peptides (Figure 2(a)), and (b) periodically replicated infinite fibril structures (Figure 2(b)).

The finite $A \beta$ amyloid protofilament segments consist of two peptides $\times$ four layers $=$ eight $A \beta_{9-40}$ peptides. The protofilaments are placed into rectangular simulation boxes and solvated with 9000 to $15,000{\text { TIP } 3 P^{47}}$ water molecules (see Table 1 ). We expect that for these four-layer fragments, the properties of the inner two layers will be close to those of longer fibrils. A finite fibril system with $C_{2}^{x}$ topology is shown in Figure 2(a).
Infinite protofilaments are constructed by periodic replication of segments containing two peptides $X$ four layers $=$ eight $A \beta_{9-40}$. The initial $z$-dimension of the simulation box is chosen to be commensurate with the $4.8 \AA$ spacing between hydrogen-bonded backbones of the parallel $\beta$-sheets. In an infinite-fibril simulation system such as the one depicted in Figure 2(b), the fibril structure is free to change in the $x y$-plane (i.e. normal to the fibril axis) during the MD simulation. However, because of the periodicity along the $z$-axis, a "twist" around the fibril axis is not possible.

\section{Conformational stability of $A \beta$-peptide protofilaments}

Our MD simulations show that the strong hydrophobic interactions between the non-polar residues of the $\beta$-strand regions lead to the formation of a well-packed core, an important factor for the stability of $\beta$-amyloid fibrils. Figures 1 , 2(c), and 3 show peptide structures at the end of 10 ns MD simulations for both finite (Figures 1 and 2(c)) and infinite periodic (Figure 3 ) fibril segments. We find that the fibril configurations remain compact for all four topologies: $C_{2}^{x}, C_{2}^{z}, F C_{2}^{x}$ and
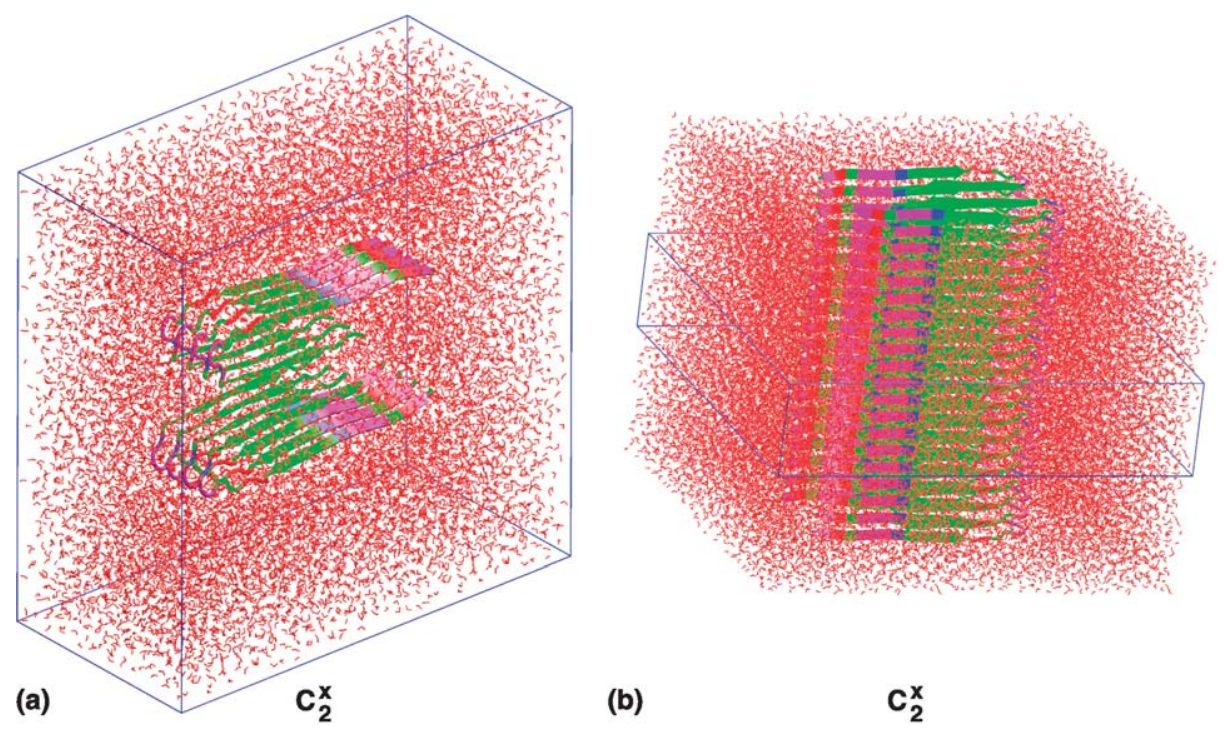

(b)

$\mathrm{c}_{2}^{\mathbf{x}}$

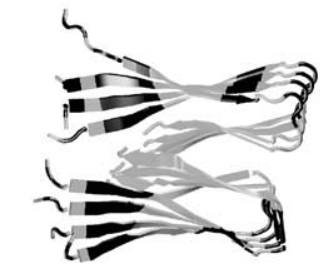

(c)

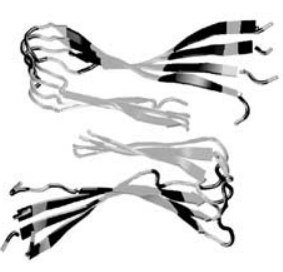

$\mathrm{C}_{2}^{\mathrm{z}}$

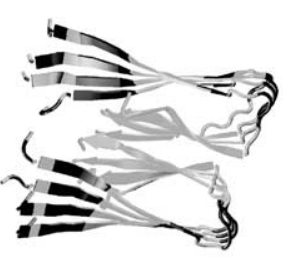

$\mathrm{FC} \underset{2}{\mathrm{x}}$

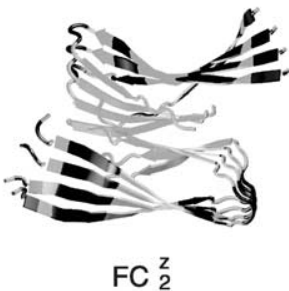

$\mathrm{FC}_{2}^{\mathrm{z}}$

Figure 2. Initial configurations of the $A \beta_{9-40}$ fibril model with $C_{2}^{x}$ symmetry for (a) the "finite" fibril segment, and (b) the "infinite" fibril system. The peptide backbones (ribbons) are shown together with the surrounding water molecules. Simulation boxes are shown as blue lines. (c) Ribbon representations of the final configurations of the finite $\mathrm{A} \beta_{9-40}$ fibril segments $C_{2}^{x}, C_{2}^{z}, F C_{2}^{x}$, and $F C_{2}^{z}$. In all cases, the fibril segments remain in compact conformations during the 10 ns simulations. 
(a)
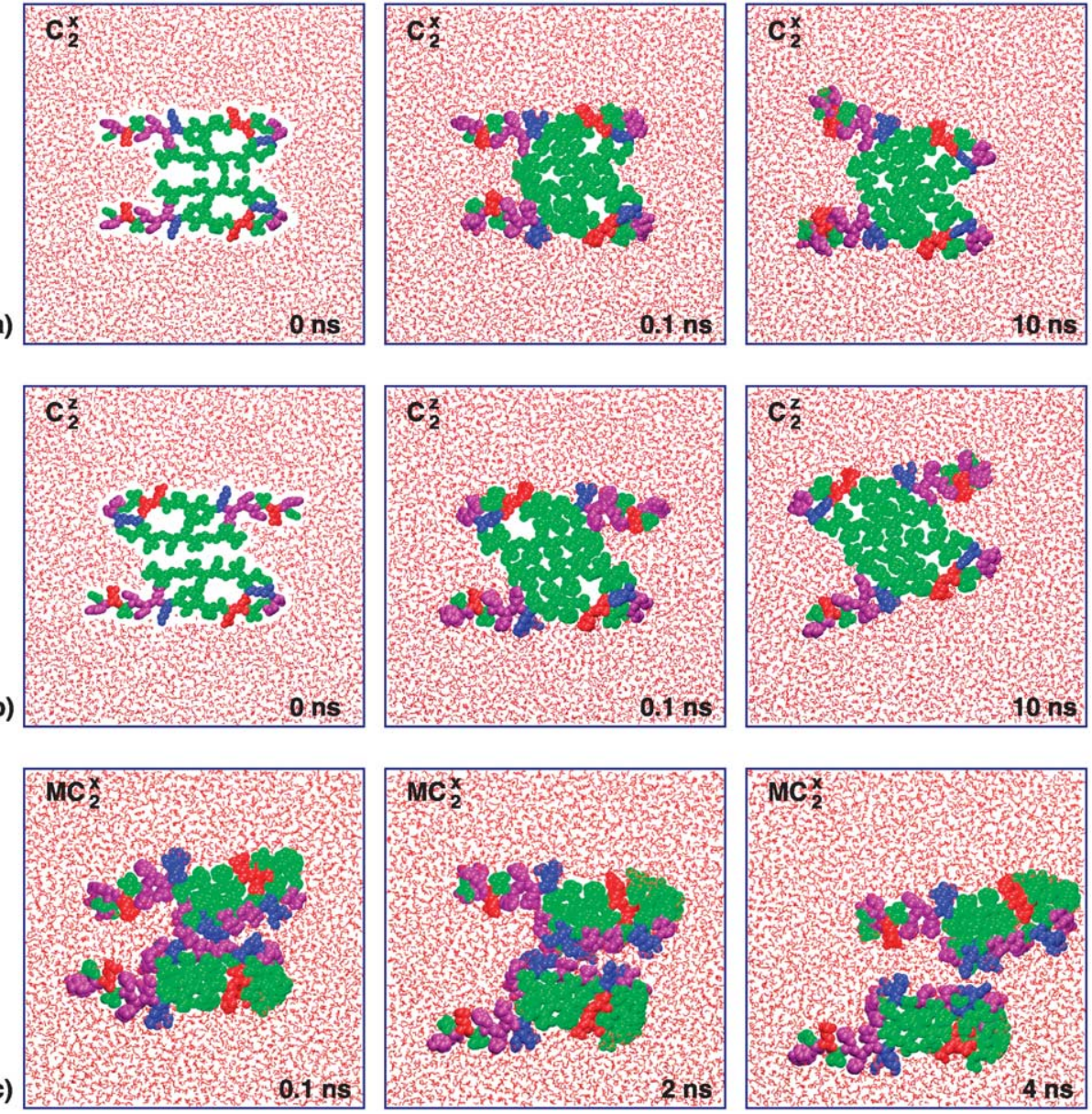

Figure 3. Snapshots of initial (0 ns), intermediate $(0.1 \mathrm{~ns})$ and final (10 ns) configurations of MD simulations of infinitely long fibril structures. (a) Top views (along the $z$ axis) are shown for $A \beta_{9-40}$ systems with $C_{2}^{x}\left((\mathrm{a})\right.$ and (c)) and $C_{2}^{z}$ symmetry (b). The system shown in (c) corresponds to a mutated sequence $\left(M C_{2}^{x}\right)$, with a hydrophilic and charged interface. The structural stability of the test $M C_{2}^{x}$ system is lost after just $\approx 2$ ns of MD simulations.

$F C_{2}^{z}$. For $F C_{2}^{x}$, we performed additional simulations with different types of force fields (Table 1), and again the wild-type $\mathrm{A} \beta_{9-40}$ fibrils with $F C_{2}^{x}$ structures are stable in all cases.

The perfect $C_{2}$ symmetries of the initial $A \beta_{9-40}$ oligomer structures are lost during the $\mathrm{MD}$ simulations. Loss of symmetry in the $C_{2}^{x}$ and $F C_{2}^{x}$ structures is more pronounced than in the $C_{2}^{z}$ and $F C_{2}^{z}$ structures. Shifting of side-chain-side-chain contacts along the $x$-axis in the $C_{2}^{x}$ and $F C_{2}^{x}$ simulations places side-chains at the dimer interface (e.g. side-chains of I32, L34, and V35 in the $C_{2}^{x}$ case, side-chains of I31, M35, and V39 in the $F C_{2}^{x}$ case) in two qualitatively inequivalent environments (see Figure 1(a) and (c)). One might expect this existence of two inequivalent environments to produce a splitting of solid state ${ }^{13} \mathrm{C}$ NMR signals into two equal-intensity components. Such a splitting has not been observed experimentally, ${ }^{4}$ suggesting that the $C_{2}^{z}$ or $F C_{2}^{z}$ structure in these simulations may correspond more closely to the $\mathrm{A} \beta_{1-40}$ fibril structure characterized in recent solid state NMR experiments.

As a test of the time-scale on which we could expect a conformational instability to become noticeable, we perform an MD simulation for a mutated sequence in the $C_{2}^{x}$ conformation, GYEVHHQKLVFFAEDVGGGGGNIKGNMNGKVN (the underlined residues are mutate $\bar{d})$. The results for this $M C_{2}^{x}$ simulation ( $M$ stands for mutation) are illustrated in Figure 3(c). The I32K and G38K mutations result in strong electrostatic repulsions between the peptides in the dimeric layer that destabilize the intermolecular $\beta$-sheet interface. As illustrated in Figure 3(c), the two protofilament strands separate during the first few nanoseconds. At $4 \mathrm{~ns}$, the intermolecular interface is fully hydrated. The observed protofilament disintegration for a mutated sequence serves as an indication that a strong conformational instability of the fibril structures studied here could become evident in $10 \mathrm{~ns}$ MD runs.

Further evidence for the structural stability of the wild-type fibrils comes from analyzing the variation of several structural parameters along the MD simulation trajectories, such as: (a) root-meansquare distances (RMSD) for the backbone $\mathrm{C}^{\alpha}$ atoms along the trajectory, and (b) Asp-Lys 
distances related to the integrity of interior D23-K28 salt-bridges.

\section{$R M S D$ values for backbone $C^{\alpha}$}

Figure 4 shows RMSD curves for the backbone $\mathrm{C}^{\alpha}$ atoms, calculated for finite $F C_{2}^{x}$ fibrils. These results are representative for the other topologies (i.e., $C_{2}^{x}$, $C_{2}^{z}$, and $F C_{2}^{z}$ ). The RMSD values are calculated by comparing the conformations at different times during the simulations to reference structures corresponding to initial $\left(\mathrm{RMSD}_{0}\right)$, final (i.e. after $\left.10 \mathrm{~ns}, \mathrm{RMSD}_{10}\right)$, and average conformations $\left(R M S D_{\text {ave }}\right)$, respectively. The average structure is obtained from fibril coordinates of the second half of the trajectory (i.e. 5-10 ns) using recursively optimal rigid-body alignment followed by averaging until convergence is achieved. As shown in Figure 4(a), during the first nanosecond of the MD trajectories, the fibril structure relaxes away from the initial model. After about $1 \mathrm{~ns}$, the $C^{\alpha}$ backbone RMSD settles at $\sim 3.5 \AA$ with respect to
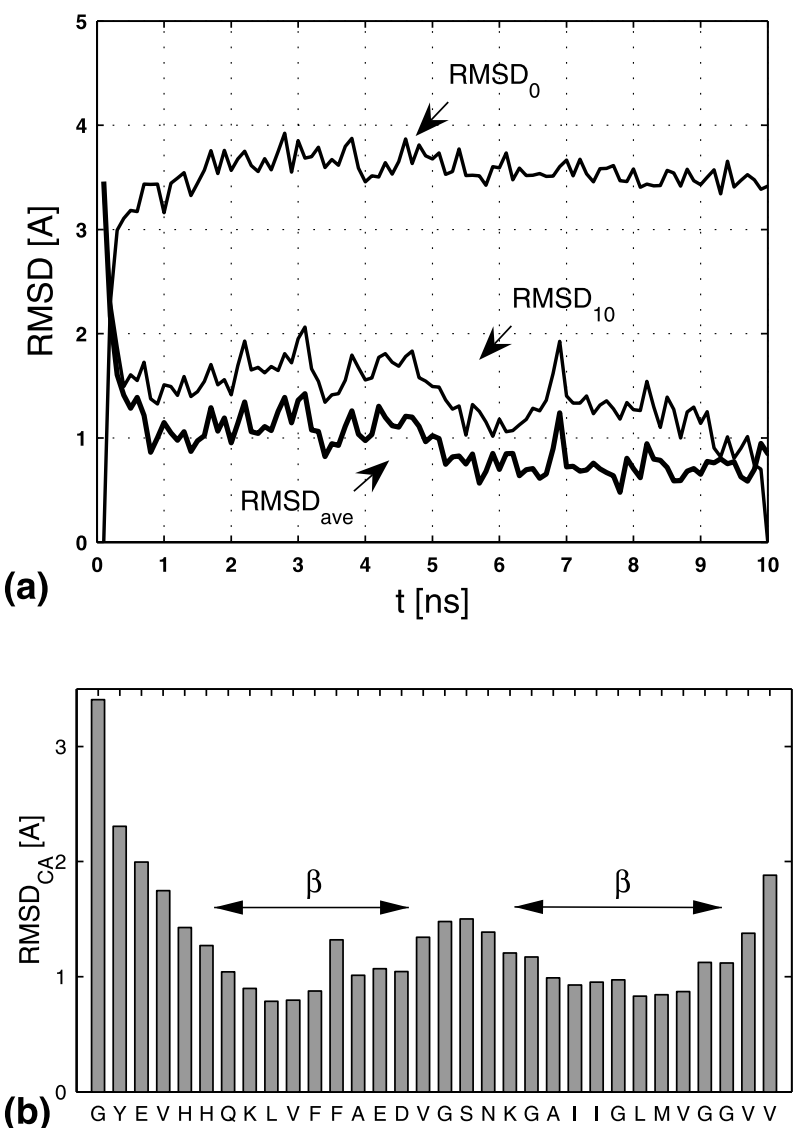

Figure 4. (a) Backbone $\mathrm{C}^{\alpha}$ RMSD deviations from the starting $\left(\mathrm{RMSD}_{0}\right)$, final $\left(\mathrm{RMSD}_{10}\right)$ and average $\left(\mathrm{RMSD}_{\text {ave, }}\right.$ 5-10 ns) structures. The RMSD curves are shown for the $F C_{2}^{x}$ system and are representative for the other cases $\left(C_{2}^{x}\right.$, $C_{2}^{z}$, and $F C_{2}^{z}$ ). (b) Local dynamical variations in the positions of the $\mathrm{C}^{\alpha}$ atoms of the amyloid monomers as measured by the RMSD from their average values over the 5 to $10 \mathrm{~ns}$ trajectory segment of the $F C_{2}^{x}$ fibril. In the structural alignment, all $C^{\alpha}$ were used. the starting structure, and $\sim 1-2 \AA$ with respect to the final structure. These RMSD curves show that during the MD simulations the protofilaments assume a stable conformation with only minor changes of their core structure occurring during the final $5 \mathrm{~ns}$ of the MD trajectories. The major structural changes in the first $1 \mathrm{~ns}$ period are interdigitation of hydrophobic side-chains between $\beta$-sheets and twisting about the $z$-axis.

Figure 4(b) shows the local dynamical variations of the $F C_{2}^{x}$ peptide monomer backbone as measured by the RMSDs of individual $\mathrm{C}^{\alpha}$ atoms from their average positions over the second half of the $10 \mathrm{~ns}$ trajectory. We find that the backbone atoms in the $\beta$-sheet regions exhibit significantly smaller fluctuations from their average positions than either the $\mathrm{N}$ or $\mathrm{C}$ termini, or the connecting loop segment.

Based on previous experimental observations that the N-terminal regions are disordered, and not likely to be part of the H-bonded $\beta$-sheet structure in the fibril, ${ }^{10,25,45,46}$ we did not include residues 1-8 of the full $A \beta_{1-40}$ sequence in our simulations. However, as shown in Figure 4(b), the remaining $\mathrm{N}$-terminal residues exhibit larger fluctuations, indicating that, indeed, they can be a part of the transition between a structured $\beta$-sheet region and a flexible $\mathrm{N}$-terminal segment.

Experimental ${ }^{13} \mathrm{C}$ NMR linewidths ${ }^{4,10}$ for most carbonyl, $\alpha$-carbon, and $\beta$-carbon sites in residues 10-39 are less than $2.5 \mathrm{ppm}$, indicating an ordered conformation in a non-crystalline environment. Linewidths for A2, D7, S8, and G9 are significantly larger, indicating structural disorder in the $\mathrm{N}$-terminal segment. In addition, linewidths for certain sites in residues $14-16$ exceed $3 \mathrm{ppm}$, suggesting local disorder around Q15. Some, but not all, sites in residues $35-39$ exhibit ${ }^{13} \mathrm{C}$ NMR linewidths in the range of 3-4 ppm. However, measurements of intermolecular carbon-carbon distances and ${ }^{13} \mathrm{C}$ NMR chemical shifts ${ }^{10,14}$ indicate that V39 participates in the parallel $\beta$-sheet structure of $A \beta_{1-40}$ fibrils. Electron paramagnetic resonance measurements on spin-labeled $A \beta_{1-40}$ and $A \beta_{1-42}$ fibrils $^{22}$ show immobilization and intermolecular dipole-dipole couplings for residues 14-38 (no data reported for residues 11-13 or V39). Thus, the experimental data indicate that the ordered structure in $A \beta_{1-40}$ fibrils extends to the $C$ terminus, in agreement with the observation in our simulations that the intermolecular hydrogen bonds in the parallel $\beta$-sheets remain intact for the $\mathrm{C}$-terminal residues.

Recent hydrogen-deuterium exchange experiments on $A \beta_{1-40}$ fibrils ${ }^{48}$ show that residues 37-39 are less strongly protected from exchange than certain other residues in the most highly ordered segments of the fibrils. However, the exchange period employed in these experiments $(25 \mathrm{~h}$ at $\mathrm{pH} 7.5$ ) is long enough to permit significant hydrogen-deuterium exchange even in structurally ordered $\beta$-sheets, so these data do not contradict the results of our simulations and of solid state NMR experiments. 


\section{D23-K28 salt bridge}

Both solid state NMR measurements ${ }^{4,10}$ and the MD simulations reported by Ma \& and Nussinov ${ }^{30}$ have identified an interior salt-bridge between D23 and K28 as an important element of the fibril structure. Figure 5(a) shows the relative arrangements of D23 (red) and K28 (blue) at the end of the $10 \mathrm{~ns} F C_{2}^{x}$ simulation of a finite fibril segment. We find that the positive K28 and negative D23 sidechains alternate along the fibril axis, with tight saltbridges both intramolecular, and between adjacent peptide layers. The time evolution of the distances (shown as green lines in Figure 5(a)) between the $\mathrm{C}^{\gamma}$ atom of the D23 residue and the $\mathrm{N}^{\xi}$ atom of $\mathrm{K} 28$, is shown in Figure 5(b). For the five salt-bridges in the interior layers of the protofilament segment, the distances fluctuate little around their average value of $3.46 \AA$, with a standard deviation of $0.24 \AA$, as shown by the histogram in Figure 5(b) for the $F C_{2}^{x}$ topology. The values of the corresponding D23K28 distances and their histograms for all four topologies simulated here are similar to the $F C_{2}^{x}$ case.

\section{Backbone and side-chain torsion angles}

The backbone and side-chain torsion angles are important structural parameters, directly related to the local secondary structure and conformational details of $A \beta$ peptides in protofilaments. Values of the residue-specific $\Phi$ and $\Psi$ angles for $A \beta_{1-40}$ fibrils have been estimated from solid state ${ }^{13} \mathrm{C}$ NMR chemical shifts determined from two-dimensional NMR spectra of samples with multiple uniformly ${ }^{13} \mathrm{C}$-labeled residues, recorded under magic-angle spinning ${ }^{4,10}$ and from solid state NMR measurements that directly probe the distances between and the relative orientations of sequential backbone carbonyl groups in samples with selectively ${ }^{13}$ C-labeled carbonyl sites. ${ }^{21}$

From MD simulations, we extract statistical values of the residue-specific backbone torsion angles $(\Phi$ and $\Psi)$ and of the side-chain angle $\chi_{1}$ for finite $A \beta_{9-40}$ fibril segments. The MD-derived mean values of the $\Phi, \Psi$ and $\chi_{1}$ angles for the three largest clusters of conformations (i.e. corresponding to the largest populations $P(\Phi, \Psi)$ and $\left.P\left(\chi_{1}\right)\right)$ are compared to values estimated using the TALOS program $^{49}$ from solid-state NMR data.

For residues in $\beta$-strand segments, the dihedral angles extracted from the $10 \mathrm{~ns} \mathrm{MD}$ trajectories of the four (i.e. $C_{2}^{x}, C_{2}^{z}, F C_{2}^{x}$ and $F C_{2}^{z}$ ) configurations are very close to the corresponding experimental estimates. For $F C_{2}^{x}$, we note a high degree of disorder at the $C$ terminus. Interestingly, only $F C_{2}^{z}$ shows two occupied $(\Phi, \Psi)$ values for residue Q15, in agreement with the experimentally observed local enhancement in the $\mathrm{CO}, \mathrm{C}^{\alpha}$ and $\mathrm{C}^{\beta}$ linewidths for residues $14-16 .{ }^{4}$ Enhanced linewidths are also observed for residues $\mathrm{H} 14$ and K16, but the MD data shows multiple values only for the $\chi_{1}$ angle at those locations.

For some residues in the loop segment, multiple dihedral-angle states with significant population are observed in the simulations, some of which are

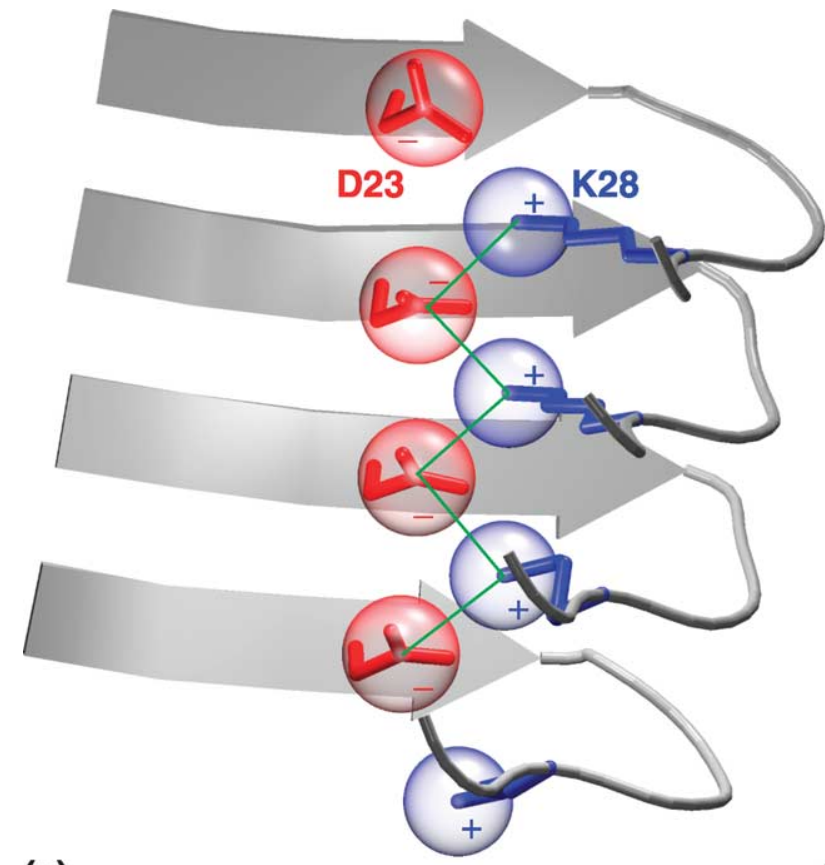

(a)

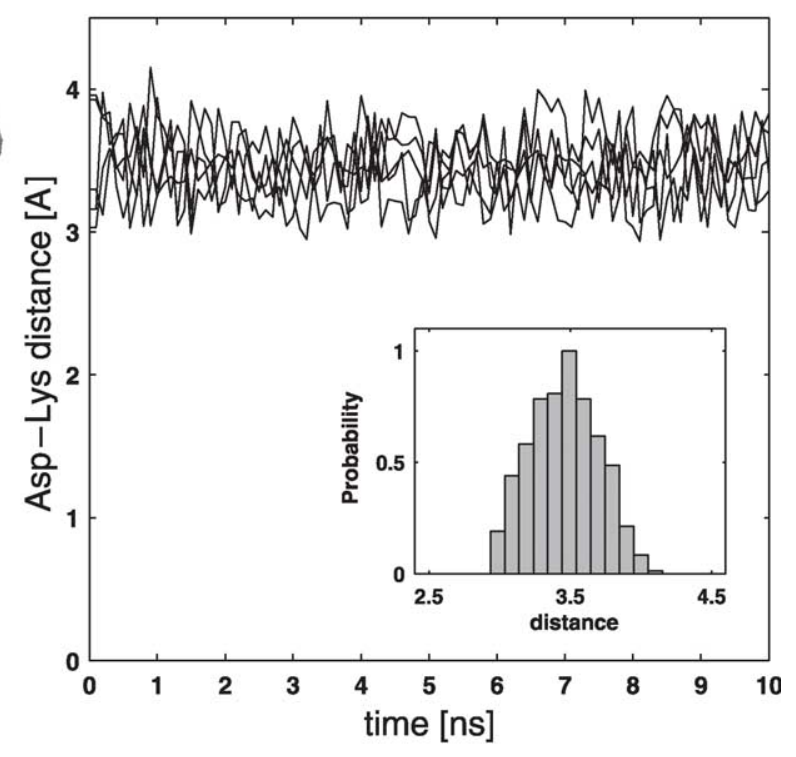

(b)

Figure 5. (a) Structure and (b) dynamics of the salt-bridge D23-K28. For clarity, only the A21-G29 region of the backbone is shown. The distances (green lines) shown in (a) are between the atoms $\mathrm{C}^{\gamma}$ of D23 and $\mathrm{N}^{\zeta}$ of K28. Their values over $10 \mathrm{~ns}$ MD trajectories (b) indicate that both intra and inter-molecular salt-bridges are equally present in the fibril structure. The inset in (b) shows a histogram of these values. 
close to the experimental estimates. Considering the generally broad distributions of torsional angles in the Ramachandran plane, the observed agreement between the average $\Phi$ and $\Psi$ values from experiments and simulations is excellent in a majority of cases. This is illustrated, for example, by the secondary structure elements depicted in Figure 2(c) for the final configurations of finite fibril segments. The only significant deviations between simulation and experiment are at residue S26, for both the CHARMM27 and AMBER94 force fields.

\section{Reconstruction of long fibrils}

At a coarse level, the structure of a perfectly ordered fibril can be described by a "rigid-body" symmetry transformation that relates successive dimeric layers in the fibril (i.e. a finite rotation followed by a translation, see Methods). If the rotation component is significant, the fibril structure adopts a helical twist. If not, the translational distance between the centers of successive dimeric layers is the only geometrical parameter needed to characterize the relative position of molecules in different layers.

A goal of our MD simulations of finite fibril segments is to extract this rigid-body transformation. For the infinite fibril system, we can only estimate the interlayer spacing, since the periodic boundary conditions preclude twisting around the fibril axis. However, the finite fibril segments are fully surrounded by a large solvation layer, allowing us to estimate the translation and rotation components of the rigid-body symmetry transformation.

To extract the rigid-body fibril transformation from the MD simulations, we need to define a subset of atoms that form a "fibril core". We consider two such definitions; (1) $\mathrm{C}^{\alpha}$ atoms of residues 15-22 and 31-38 forming the $\beta$-strand regions, and (2) $C^{\alpha}$ atoms of residues $22-29$ at the loop. These "core" definitions are used both for individual peptides ("monomer-based" reconstruction) and simultaneously for two peptides in a single layer ("dimer-based" reconstruction).

With the $C^{\alpha}$ atoms of residues $15-22$ and 31-38 used as the core, the rotation $(\phi)$ and translation $(t)$ axes of the rigid-body transformation relating the peptide layers have noticeably different directions. A long $A \beta_{9-40}$ protofilament reconstructed using these axes exhibits a twisted structure with superhelical ("phone cord"-like) appearance. In contrast, for fibril structures using residues in the loop regions (e.g. residues 22-29), the translation and rotation axes are almost parallel with each other and normal to the fibril layers. Moreover, the RMSD values, as measures of deviations from ideal fibril symmetry, are smaller. Therefore, considering the straight appearance of $A \beta_{1-40}$ protofilaments in transmission electron microscopy images of negatively stained fibrils grown with gentle agitation, we use the $C^{\alpha}$ atoms of loop residues 22-29 to define the fibril core region and to extract fibril parameters. These differences between fibril parameters extracted using strand and loop regions, are a consequence of two competing effects; (1) the formation of twisted $\beta$-sheets along the fibril axis by the residues in the $\beta$-strand regions, and (2) the straight structure of the U-shaped loop region. For the simulations of short fibril segments, these two opposing effects result in structures that are relatively straight at the loops, but twisted at the $\mathrm{N}$ and $C$-terminal $\beta$-strands. These competing effects are also apparent when we compare the fibril reconstructions using monomers and dimers of peptides.

For monomer-based reconstructions, the angle between the resulting two fibril axes corresponds to the angle between the two strands of the protofilaments. The values are about $30^{\circ}$ or larger for all cases, except $F C_{2}^{x}$. Clearly, four layers of two peptides each are not sufficient to mimic an infinite fibril, with competing forces breaking the fibril symmetry. Only in the case of the $F C_{2}^{x}$ topology do

Table 2. Symmetry transformation parameters of $A \beta_{9-40}$ fibrils extracted from MD simulations of finite fibril segments, and mass-per-length values of corresponding reconstructed long $A \beta_{9-40}$ and $A \beta_{1-40}$ protofilaments

\begin{tabular}{|c|c|c|c|c|c|c|c|}
\hline & $F C_{2}^{x}$-dimer & $F C_{2}^{x}$ & $C_{2}^{x}$ & $F C_{2}^{z}$ & $C_{2}^{z}$ & $F C_{2}^{x}-\mathrm{A}$ & $F C_{2}^{x}-\mathrm{AGS}$ \\
\hline \multicolumn{8}{|c|}{ Representative structure (ns) } \\
\hline & 7.8 & 7.8 & 9.1 & 7.6 & 8.9 & 7.5 & 7.1 \\
\hline \multicolumn{8}{|c|}{$\phi=\mathbf{t}$, core region composed of $C^{\alpha}$ for residues $22-29$} \\
\hline$t_{x}\left(\AA_{0}\right)$ & 0.186 & -0.198 & -1.372 & -0.682 & 0.890 & -0.628 & 0.707 \\
\hline$t_{y}(\AA)$ & -0.858 & -1.104 & -0.883 & 3.221 & -0.833 & 0.397 & -0.732 \\
\hline$t_{z}(\AA ⿻)$ & 4.763 & 4.688 & 4.617 & 3.454 & 4.746 & 4.846 & 4.750 \\
\hline$|t|(\AA)$ & 4.843 & 4.820 & 4.897 & 4.772 & 4.900 & 4.903 & 4.858 \\
\hline$|\phi|$ (deg.) & 1.51 & 0.72 & 1.04 & 1.31 & 2.39 & 3.60 & 0.18 \\
\hline \multicolumn{8}{|c|}{ Mass-per-length (kDa/nm) } \\
\hline MPL for $A \beta_{9-40}$ & 13.8 & 13.9 & 13.7 & 14.1 & 13.7 & 13.7 & 13.8 \\
\hline MPL for $A \beta_{1-40}$ & 17.8 & 17.9 & 17.6 & 18.1 & 17.6 & 17.6 & 17.7 \\
\hline \multicolumn{8}{|c|}{ Percentage hydrophobic area } \\
\hline $\mathrm{ASA}_{\mathrm{H}}(\%)$ & 60 & 55 & 55 & 55 & 53 & 57 & 54 \\
\hline
\end{tabular}

Residues 22-29 near the loop segment of $\mathrm{A} \beta_{9-40}$ monomers define the core regions. $F C_{2}^{x}$ - dimer lists parameters for the corresponding dimeric layer. The predicted fraction of hydrophobic, lateral accessible surface area $\left(\mathrm{ASA}_{\mathrm{H}}\right)$ of corresponding long fibrils is also reported for each case. 
we get sufficiently consistent results to extract that symmetry with confidence. This suggests that the finite fibrils with $C_{2}^{x}, C_{2}^{z}$, and $F C_{2}^{z}$ topology do not strictly conform to fibril symmetry, and indeed monomer and dimer-based reconstruction results in substantially different structures of long protofilaments. For these topologies, we thus report only monomer-based reconstruction results.

Table 2 lists values of the symmetry transformation parameters of $A \beta$ amyloid fibrils extracted from MD simulations of finite fibril segments. All results are obtained with residues 22-29 defining the fibril core. In that case, the translation and rotation axes are almost parallel with each other. We find that imposing this condition leads to values of the RMSD between the superimposed coordinates that are nearly as good as those of an unrestrained superposition, despite having reduced the number of free parameters from 6 to 4 . For example, the RMSD between the core regions for overlapping the middle layers of the $F C_{2}^{x}$-dimer structure in the general case is $0.635 \AA$, which is not significantly smaller than the RMSD value $0.642 \AA$, obtained if the two axes are constrained to have the same direction. Therefore, all the cases presented here (and the corresponding parameters in Table 2) have the rotation and translation axes coincident.

Figure 6(b) shows values of the translation distance $(|t|$, upper continuous line) and rotation angle ( $\phi$, lower continuous and dotted lines) extracted for the dimer transformation from the finite fibril segment simulation of the $A \beta_{9-40} F C_{2}^{x}$ system. The dotted curve corresponds to values extracted by using the optimal rigid-body alignment (with no constraint on the rotation axis), while the continuous line shows values extracted by considering the optimal rotation about the translation axis $t$. After a rapid initial equilibration (i.e. 2-3 ns) at the beginning of the simulations, the structural parameters of the fibrils fluctuate around their average values without significant drift. For all finite fibril segments, we observe left-handed twisting of the A $\beta$-fibrils (see Table 2), consistent with experimental observations. ${ }^{27}$ The rotation angle per peptide length is about $1-3^{\circ}$, corresponding to about 120-360 peptide layers (50-200 nm) per full turn. Experimental helical pitch values, as observed in the apparent twist periodicity of electron microscope images, are of the same order of magnitude for $A \beta_{1-40}$ and $A \beta_{10-35}$ fibrils., 42,27 Interestingly, $A \beta_{1-40}$ fibrils with molecular structures similar to the structural models treated here do not have a clearly resolved twist in electron microscopy images, ${ }^{4}$ consistent with our simulation-derived models (e.g. see data in Table 2 and the reconstructed fibrils in Figure 7).

Based on the symmetry transformation parameters shown in Table 2, we reconstructed long amyloid fibrils. Figure 7 shows fibril models built using the $F C_{2}^{x}$ parameters, at detail levels ranging from atomistic (Figure 7(a)) to coarse-grained (i.e. with $A \beta_{9-40}$ molecules represented by rigid boxes centered at the geometric center of the core regions,
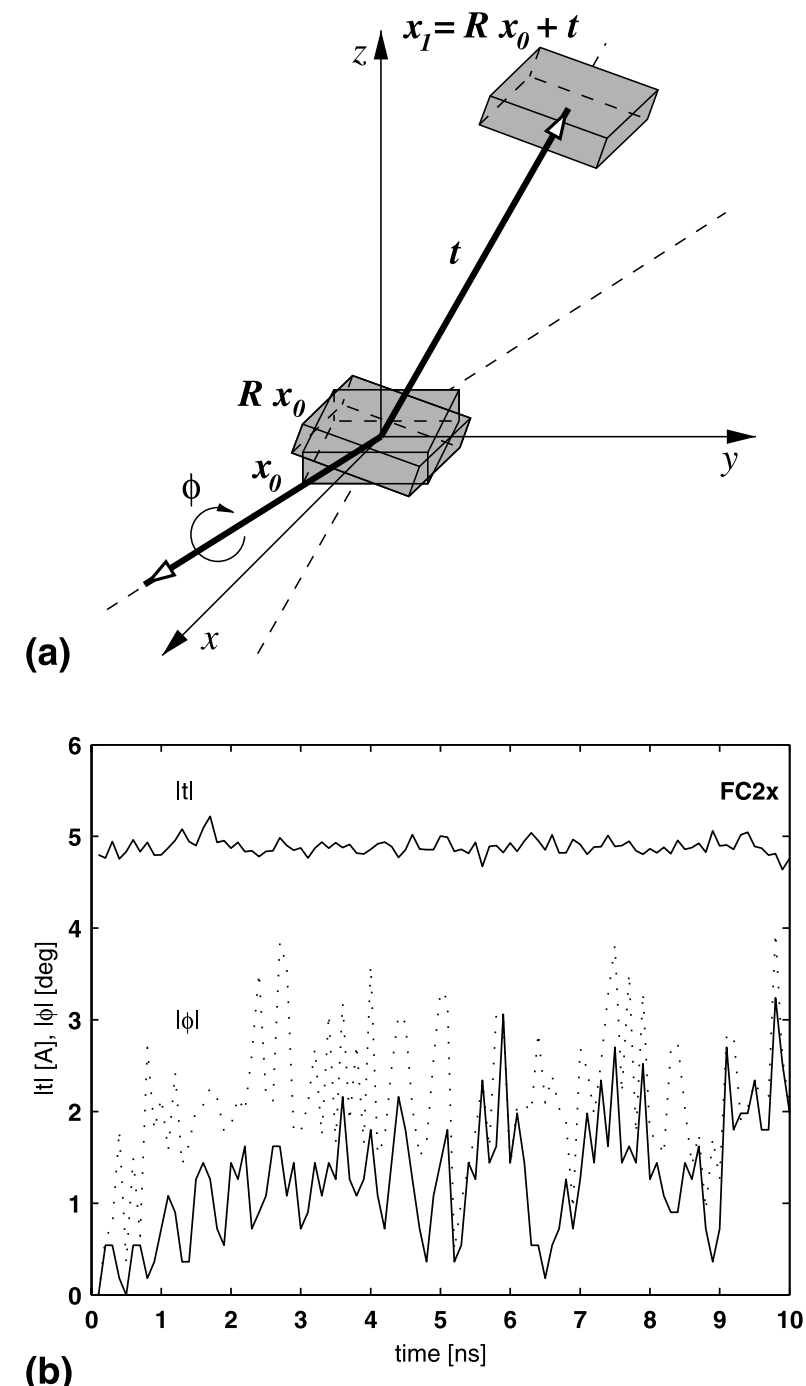

Figure 6. Reconstruction of long fibrils. (a) Schematic representation of the rigid-body transformation relating consecutive $A \beta_{9-40}$ dimeric layers in the twisted fibril models. (b) Values of the translation distance $(|t|$, upper continuous line) and rotation angle $(\phi$, lower continuous and dotted lines) extracted using the dimer transformation from the finite-fibril simulation of the $A \beta_{9-40} F C_{2}^{x}$ system. The dotted curve corresponds to values from optimal superposition. The continuous line shows values extracted by restricting the rotation axis to be parallel with the translation axis.

Figure 7(b)). In reconstructing the detailed all-atom models, we use the actual coordinates of an $A \beta_{9-40}$ molecular layer (i.e. a dimer structure) that corresponds to a "representative" instantaneous structure from the MD trajectory, with the smallest RMSD to the average conformation of the final $5 \mathrm{~ns}$. These representative coordinates are then translated and rotated recursively (see Methods) using the average values of the symmetry transformation parameters extracted from the MD trajectories (Table 2) for $F C_{2}^{x}$-dimer. Using the atomically detailed MD data, our fibril reconstruction method permits the generation of large-scale all-atom fibril 
(a)

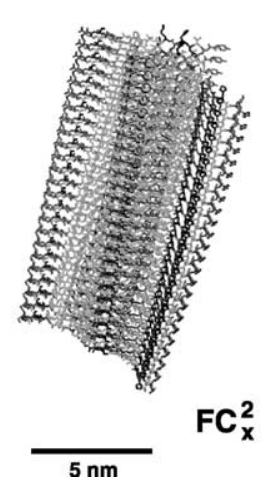

(b)

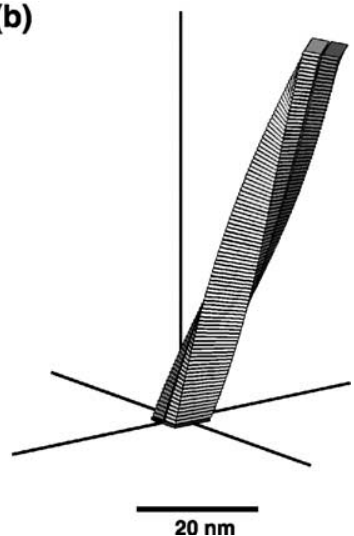

Figure 7. (a) Atomic level, and (b) coarse structural models of long $A \beta$ fibrils with $F C_{2}^{x}$ topology. In the schematic fibril model (b), each $A \beta$ peptide monomer is represented by a rectangular block. Distance scales are indicated as bars. In this reconstruction, the translation and rotation axes have the same direction.

structures. These MD-based, predicted structures for long fibrils can be compared directly with largescale fibril characteristics observed experimentally (i.e. mass-per-length estimates, X-ray diffraction, hydrophobic properties of molecular fibril surfaces, etc.), as shown next.

\section{Mass-per-length of amyloid fibrils}

The mass of the monomeric $A \beta_{1-40}$ peptide is $4.32 \mathrm{kDa}$. Therefore, in ideal $\beta$-sheet structural models where dimeric layers are simply replicated along their $z$ axis, separated by an average distance dictated by the backbone hydrogen bond length of 4.7-4.8 $\AA$, the expected MPL value is about 18.0$18.4 \mathrm{kDa} / \mathrm{nm} .{ }^{4,10,12}$ As shown in Methods, MPL values are inversely proportional to the projection of the translation distance vector $\mathbf{t}$ onto the fibril axis $\phi$. Values of $18-18.4 \mathrm{kDa} / \mathrm{nm}$ are, therefore, lower limits of the MPL values for dimeric $A \beta$ fibrils. By using equation (4) and an angle of only $20^{\circ}$ or $30^{\circ}$ between the translation and rotation axis, the estimates for average MPLs could be as much as 21-22 kDa/nm. However, as an immediate consequence, the fibril would form a "phone cord"-like superhelix, which has not been seen in electron microscopy experiments. More evidence for MPL values near $18-18.4 \mathrm{kDa} / \mathrm{nm}$ comes from the following analysis of X-ray fiber diffraction data.

\section{$X$-ray fiber pattern of fibril segments and long amyloid fibrils}

A principal, defining feature of $A \beta$ amyloid fibrils, distinguishing them from other types of protein fibrils, is the observation of a cross- $\beta$ X-ray fiber diffraction pattern, with $4.7-4.8 \AA$ and $8-10 \AA$ reflections in the meridional and equatorial directions, respectively. ${ }^{1,50}$ Here, we calculate $X$-ray fiber

diffraction patterns from the MD simulation structures.

Figure 8(a) shows diffraction patterns computed from instantaneous MD structures of finite fibril segments in the four topologies investigated here: $C_{2}^{x}, C_{2}^{z}, F C_{2}^{x}$ and $F C_{2}^{z}$. Because of symmetry, only a single quadrant is shown for each case (see Methods). In all four cases, the fingerprint of the cross- $\beta$ pattern (i.e., the meridional reflections at $\sim 4.4-5.0 \AA$ and the accompanying equatorial reflections at $\sim 9.8-11.2 \AA$ ) is present. ${ }^{9,50}$ Note that Figure $8(a)$ is a $Q$-weighted reduced scattering representation of the diffraction pattern (i.e. $Q i(Q)$,
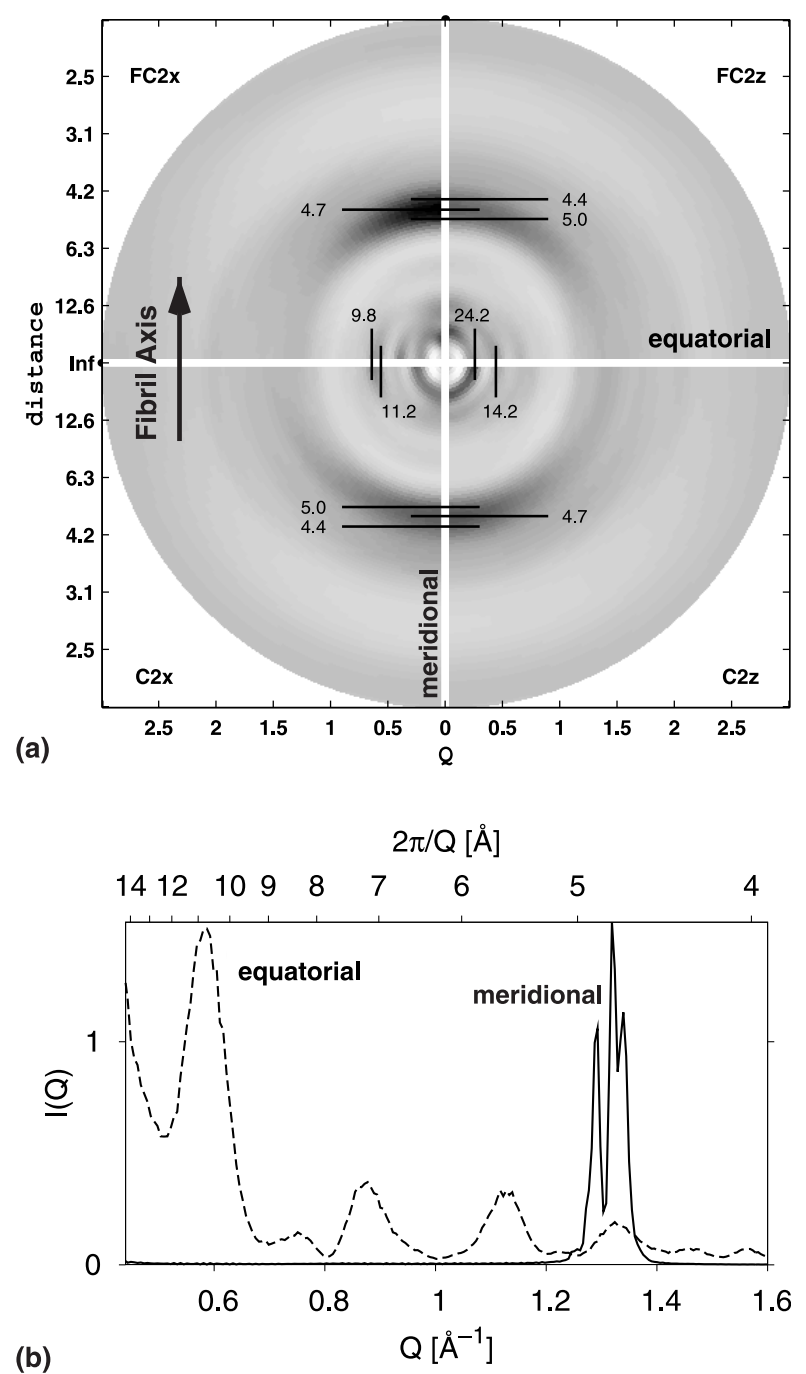

Figure 8. (a) X-ray fiber diffraction patterns calculated from MD simulations of all-atom finite $A \beta_{9-40}$ protofilament segments with four dimeric layers and $C_{2}^{x}, F C_{2}^{x}, C_{2}^{z}$ and $F C_{2}^{z}$ topologies, respectively. Only one quadrant is shown for each topology. $Q$ and distances are in units of $\AA^{-1}$ and $\AA$, respectively. (b) The calculated meridional and equatorial X-ray fiber diffraction patterns (arbitrary scale) are shown for a long, reconstructed $F C_{2}^{x}$ protofilament (corresponding to the structural model depicted in Figure 7). The parameters used in reconstructing the fibril were extracted from relative positions of dimeric layers in the MD simulations. 
see Methods). Interestingly, even though these scattering patterns are calculated for instantaneous MD structures of short segments (i.e. only four layers) of $A \beta$ protofilaments, one or two low-angle equatorial peaks are present besides the reflection at $\approx 10 \AA$ (Figure 8(a)). While the finite fibril size in these calculations prevents us from drawing conclusions regarding the quantitative aspects such as the relative peak intensities in these scattering patterns, they do allow a comparison with experimental data in terms of numbers and positions of the observed peaks. For example, the equatorial peaks present low-angle reflections similar to those observed in the experimental data, ${ }^{50}$ even though only short protofilaments are used in the calculations.

Figure 8(b) shows calculated meridional and equatorial X-ray fiber diffraction patterns for a long, reconstructed $F C_{2}^{x}$ protofilament, with the core region (residues 22-29) defined near the loop. The parameters used in reconstructing the fibril are extracted from relative positions of dimeric layers in the MD simulations (the $F C_{2}^{x}$-dimer case in Table 2). These patterns are computed directly using in-house code. In this plot, the largest meridional $(\approx 4.7 \AA)$ and equatorial $(\approx 11 \AA)$ peaks are scaled to have the same height. The relative heights of these peaks would generally depend on the choice of $B$ factors and on the range of random tilt angles of the fibrils with respect to the fibril axis. However, for clarity, in these calculations the $B$ factors and the variability of fibril axis tilt angles are not included. It is evident that, for long fibril structures, the peaks corresponding to the cross- $\beta$ motif are strongly enhanced as compared to the other reflections.

The calculated patterns in Figure 8(a) and (b) demonstrate that the protofilament structures in our MD simulations and the structural models of long, reconstructed fibrils are in qualitative agreement with experimental X-ray fiber diffraction data. More importantly, they also suggest that the equatorial reflections could be sensitive to structural differences between different molecular fibrils, such as the "flipped" (i.e. $F C_{2}^{x}, F C_{2}^{z}$ ) and "un-flipped" (i.e. $C_{2}^{x}, C_{2}^{z}$ ) topologies.

We note that the mass-per-length of the long reconstructed $A \beta_{9-40}$ protofilament shown in Figures 7 and $8(\mathrm{~b})$ is about $13.8 \mathrm{kDa} / \mathrm{nm}$, corresponding to a spacing of $4.843 \AA$ between dimeric layers. In contrast, experimental STEM measurements of MPL in $A \beta_{1-40}$ fibrils indicate a spacing of approximately $4.0 \mathrm{~A}$, projected onto the long axis of the fibrils. The experimental MPL determination is based on the quantification of intensities in STEM images of unstained $A \beta_{1-40}$ fibrils dried onto thin carbon films. These measurements are independent of structural order or disorder. Reconstructed $\mathrm{A} \beta_{9-40}$ protofilaments with MPL values similar to the experimental STEM results can be generated if the $\phi$ and $t$ axes are not parallel, but then the calculated diffraction patterns show meridional peaks near $4.0 \AA$ (in disagreement with fiber diffraction experiments). Thus, the experimental STEM data may be affected by adsorption of additional material to the $A \beta_{1-40}$ fibrils.

\section{Hydration of amyloid fibrils}

Unlike most functional proteins, peptide fibrils may not form densely packed cores. For example, Perutz et al. have proposed that polyglutamine fibrils may be water-filled nanotubes. ${ }^{51}$ X-ray fiber diffraction data have been used to support this proposal. $^{51,52}$ For the more hydrophobic A $\beta$ peptides, one may expect a more protein-like packing. Indeed, solid state ${ }^{13} \mathrm{C}$ NMR spectra of amyloid fibrils formed by $A \beta_{1-40}$ and fragments thereof are not significantly affected by the hydration level, ${ }^{19}$ indicating that no significant structural changes occur upon removal of water by extensive lyophilization. However, side-chain dynamics may be affected. In the lyophilized state, certain side-chains may be disordered statically; in the hydrated state, the side-chains are mobile, producing a motional narrowing effect in the NMR spectra. In the following, we study the packing of the $A \beta$ amyloid fibrils, characterize their molecular surface, and inspect the simulation structures for interior hydration.

\section{Accessible surface area}

We use the SurfRace program ${ }^{53}$ to estimate the solvent-accessible surface area (ASA) of reconstructed $A \beta$-amyloid fibrils. By repeating the calculation for fibrils of different lengths, we eliminate the contributions of the protofilament surfaces at the ends. In the limit of an infinitely long $\mathrm{A} \beta$ fibril in the $F C_{2}^{x}$ conformation, the hydrophobic fraction of the accessible surface area $\left(\mathrm{ASA}_{\mathrm{H}}\right)$ is about 0.6. Results for all the cases considered in this work are given in Table 2. A recent study by Lins et al. ${ }^{54}$ showed that most proteins, irrespective of their size, have roughly equal fractions of hydrophobic and hydrophilic ASA. This would suggest that the $A \beta$ fibrils present a somewhat larger hydrophobic ASA than a typical protein. To test this observation, we use the SurfRace $\operatorname{program}^{53}$ to estimate the $\mathrm{ASA}_{\mathrm{H}}$ values for a set of nonhomologous proteins. This set of 195 highresolution structures was selected from the Protein Data Bank ${ }^{55}$ by Liwo et al., ${ }^{56}$ and was subsequently used as a training set for residue-level statistical interaction potentials. ${ }^{56,57}$ As in the study by Tsodikov et al., ${ }^{53}$ we use the set of van der Waals radii from Richards ${ }^{58}$ and a probe radius of $1.4 \AA$. The distribution of $\mathrm{ASA}_{\mathrm{H}}$ values for this representative set of protein structures is shown in Figure 9. In agreement with Lins et al. ${ }^{54}$ we note that the $\mathrm{ASA}_{\mathrm{H}}$ distribution for proteins appears slightly skewed toward smaller fractions. Although the exact $\mathrm{ASA}_{\mathrm{H}}$ values may depend on the set of representative protein structures that is analyzed and on the details of the ASA calculation 


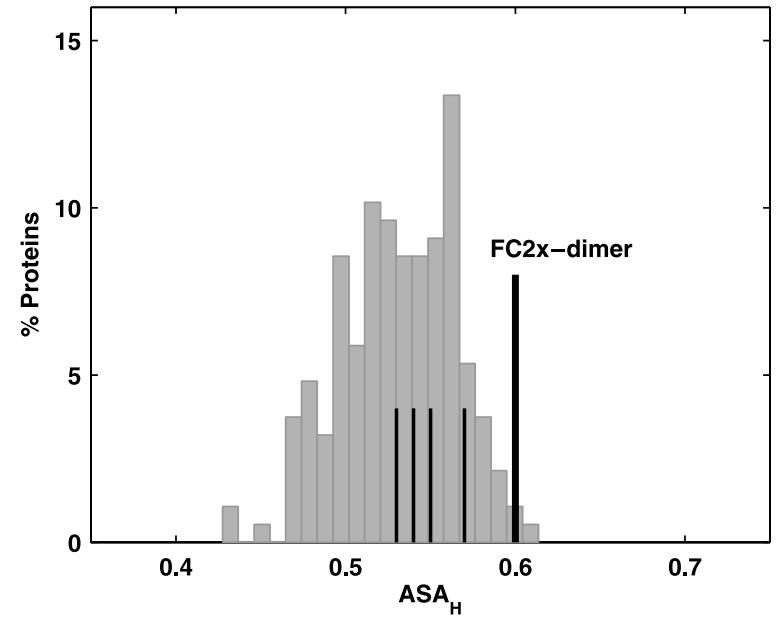

Figure 9. Fraction of hydrophobic accessible surface area $\left(\mathrm{ASA}_{\mathrm{H}}\right)$ for fibril models (black lines) and the distribution of $\mathrm{ASA}_{\mathrm{H}}$ values calculated for a representative, non-homologous set of protein structures (gray). The long thick line indicates the $F C_{2}^{x}$ system reconstructed by analyzing the full dimeric layer of the fibril. The smaller black lines correspond to the four topology types, for fibrils reconstructed using only monomers.

method, ${ }^{53,54,59}$ we observe that there is a small fraction of proteins (e.g. hemoglobin, code 1BAB) present in the PDB that have $\mathrm{ASA}_{\mathrm{H}}$ values comparable (i.e. about 0.6 ) to our fibril models. Therefore, we conclude that while they present a somewhat larger fraction of hydrophobic area than a typical protein, the $A \beta_{9-40}$ fibrils are not entirely different, having a hydrophobic character similar to the more hydrophobic structures deposited in the PDB.

The relatively high value of $A S A_{H}$ for $A \beta_{9-40}$ fibrils may also be related to the fact that the $A \beta_{9-40}$ sequence contains an unusually large proportion of hydrophobic residues $(65.6 \%$, counting glycine residues as hydrophobic). For $\mathrm{A} \beta_{1-40}$ fibrils, which contain only two additional hydrophobic residues in the disordered $\mathrm{N}$-terminal segment $(57.5 \%$ total hydrophobic residue fraction), the value of $\mathrm{ASA}_{\mathrm{H}}$ would be lower.

More importantly, the relatively high fraction of exposed hydrophobic surface area may explain the lateral aggregation of multiple protofilaments into mature $A \beta$ amyloid fibrils.

\section{Interior water channels}

In our $\mathrm{MD}$ simulations of finite $\mathrm{A} \beta$ fibril segments, we observe that internal cavities present in the starting conformation are only partially collapsed during the initial equilibration, and become hydrated by internal water molecules. The largest internal water channels are in the vicinity of the internal D23-K28 salt-bridges. In contrast, an inspection of the MD simulation trajectories for infinitely long fibrils (sample starting configuration shown in Figure 2(b)), does not reveal interior hydration. As shown in Figure 3, for infinitely long fibrils we find a compact side-chain packing during the simulations, without any significant penetration of water molecules in the fibril structure. One possible explanation would be that the initial fibril structures are relatively poorly packed, allowing water to enter into the fibril interior early in the simulations. Interior water would then prevent a further compaction of the fibril structure and water expulsion during the MD time-scale. To test this hypothesis, we ran an additional $10 \mathrm{~ns}$ finite segment simulation of the $F C_{x}^{2}$ system (with parameters listed at the bottom of Table 1), using as initial conformation the already compact and "dry" final fibril structure of a 10 ns trajectory of the infinitely long $F C_{x}^{2}$ system (see Table 1). This new starting conformation is re-solvated with TIP3P water molecules both above and below the fibril segment (as in Figure 2(a)).

In the course of the simulation, we find that water molecules gradually penetrate into the fibril structure, starting from the ends. Eventually, water occupies the same channels found before in the simulation starting from a poorly packed fibril. We conclude that the absence of interior water does occur in finite fibril segments at equilibrium, and that its absence from the infinitely long fibrils is most likely a consequence of the slow equilibration between interior water and the bulk phase, ${ }^{60,61}$ without access from the ends.

Figure 10 shows a top view (i.e. along the fibril axis) of the interior hydration of simulated $A \beta_{9-40}$ amyloid protofilaments. This illustration corresponds to the finite $F C_{2}^{x}$ segment described above, at the end of the $20 \mathrm{~ns}$ MD simulation (i.e. a $10 \mathrm{~ns}$

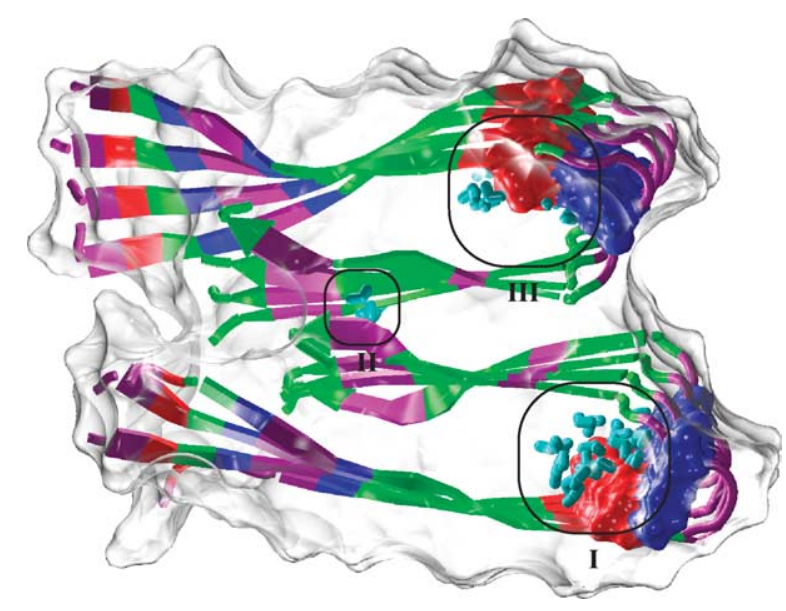

Figure 10. Interior hydration of $A \beta_{9-40}$ amyloid fibrils. Top view (i.e. along the fibril axis) of the finite $F C_{2}^{x}$ fibril segments is shown for the final structure of an MD trajectory. The secondary structure elements of the fibril are represented schematically and colored according to the residue type. Only water molecules in the three cavity regions of the fibrils are shown, colored as cyan. The molecular surface of the D23-K28 salt-bridges is also shown, using red for D23 and blue for the K28 residue. 
trajectory of a finite segment that continues another 10 ns trajectory of an infinitely long fibril, last entry in Table 1). The secondary structure elements of the fibril are represented schematically. The $F C_{2}^{x}$ protofilament is fully hydrated with 9631 water molecules, but only the water molecules in the interior cavities of the fibrils are shown. As described above, the starting conformations do not have water molecules in the interior of the fibril structure, and no significant interior hydration is observed during the $10 \mathrm{~ns}$ simulation of the infinitely long system. However, during simulations of finite fibril segments, the internal cavities are accessible from the ends of the fibrils. The largest internal hydrated cavities (marked as I and III in Figure 10) are bordered on one side by the strongly charged molecular surface formed by the D23-K28 salt-bridges, and on the other side by the hydrophobic surface of residues F19, A21, A28, I32, and L34. A water wire (denoted II in Figure 10) also appears in the vicinity of the $\mathrm{C}$-terminal residues in a narrow hydrophobic channel bordered by intermolecular G37-M35 and G37-V39 contacts, possibly related to the "glycine vents" observed by Lakdawala et al. in MD simulations of a significantly different $\mathrm{A} \beta_{10-35}$ fibril model. ${ }^{62}$ Such hydrogen-bonded water wires in sub-nanometer hydrophobic pores have been seen before in simulations of solvated carbon nanotubes. ${ }^{63}$ The hydration characteristics of the internal cavities I, II and III in the fibrils are both quantitatively and qualitatively different. The narrow channel II near the $\mathrm{C}$ terminus is entirely hydrophobic and water forms a single file arrangement. In contrast, the large, strongly hydrated internal cavities I and III are hydrophilic, and water forms an extended hydrogenbonded network.

Our simulations show that the otherwise potentially destabilizing effect of burying the charged residues D23 and K28 in the core of the parallel molecular structure may be compensated not only by stabilizing electrostatic interactions between the charged groups in a 1D ionic crystal-like arrangement (as shown in Figure 5(a)), but also by interior solvation. Recent studies ${ }^{64,65}$ showed that charged residues are important factors in the dynamics of protein aggregation and in the stability of $\beta$-sheet structures. Based on previous studies, ${ }^{66,67}$ the Oliveberg-Richardson-Richardson rule ${ }^{64}$ suggests that aggregation is generally prevented by the presence of uncompensated, inward-pointing charged residues. Our MD results show that due to the staggered intermolecular arrangement of the D23-K28 contacts, and due to interior hydration, the charges inside the core of amyloid fibrils are in fact compensated and may even be a stabilizing factor.

\section{Conclusions}

Using all-atom MD simulations in explicit solvent, we explore several possible topologies of Alzheimer's $A \beta_{9-40}$ peptide protofilaments. The initial structural models are based on recent solid state NMR experiments, ${ }^{5,10}$ and MD simulations of both "finite" segments and "infinitely long" protofilaments are performed. Four different topologies are considered for stacking the hydrophobic $\beta$-strand regions of the $A \beta_{9-40}$ molecules against each other. During the initial parts of the MD simulations, the starting configurations undergo a significant optimization of the side-chain packing that results in more compact, twisted structures of the $A \beta_{9-40}$ protofilaments. The hydrophobic regions of the fibrils remain compact in all cases and consistent with the starting parallel $\beta$-sheet structure indicated by the experimental data.

The MD simulation results demonstrate that all four topologies considered here for the wild-type $A \beta_{9-40}$ amyloid fibrils are structurally stable on a time-scale of $10-20 \mathrm{~ns}$. Such a structural polymorphism on a molecular level is a characteristic feature of amyloid fibrils. ${ }^{4}$ In contrast, corresponding models of protofilaments designed with a hydrophilic-charged intermolecular interface dissociate on a time-scale of only 2-4 ns. Overall, we find good agreement with dihedral angles estimated from solid state NMR experiments for all four topologies.

The structural analysis of MD trajectories reveals that regions near the loop segment are relatively rigid (i.e. residues E22-G29). These core regions form nearly straight regions along the fibril axis in competition with the twisted parallel $\beta$-sheets of the $C$ and $\mathrm{N}$-terminal peptide segments. For finite fibrils, this results in deviations from the symmetry of infinite fibrils.

We extract translational and rotational parameters relating adjacent molecular layers in protofilaments to reconstruct longer fibril models. However, due mainly to the finite length of the fibril segments studied here, we find that in only one case $\left(F C_{2}^{x}\right)$ both strands in the protofilament tend to have the same fibril growth axis, permitting a dimerbased analysis. In the other cases, the finite protofilament structure is sufficiently distorted to make a reliable estimate of the molecular fibril symmetry transformation difficult. The MD-based, reconstructed models of long $A \beta_{9-40}$ amyloid fibrils present a more hydrophobic accessible surface area than most proteins, yet not much different from highly hydrophobic proteins such as tetrameric hemoglobin. The presence of a relatively large fraction of exposed hydrophobic surface area $\mathrm{ASA}_{\mathrm{H}}$ could explain the experimentally observed tendency to lateral assembly of the $\mathrm{A} \beta$ protofilaments into thicker fibers (similar to the aggregation of hemoglobin in sickle cell anemia 68,69 ).

Simulations of fibril fragments show that interior hydration could play an important role in the structural stability of $A \beta$ fibrils. Unlike the large, $\beta$-helical channels suggested for other fibril-forming peptides such as polyglutamine ${ }^{70}$ or Sup $35^{52}$ 
the interior channels present in our models are smaller, appearing as either "single-file" hydrophobic channels, at the intermolecular interface along the fibril axis, or as larger, "hydrophobiccharged" cavities, near the D23-K28 salt-bridges. The effect of interior water in our models can be either to stabilize the fibril structure by hydrating interior salt-bridges in hydrophobic-charged channels, or to destabilize the lateral association of the two imperfectly packed protofilament strands with hydrophobic channels at the intermolecular interface.

Regarding the D23-K28 salt-bridge, the MD results show that the inward-pointing charged residues D23 and K28 present a stabilizing staggered intermolecular arrangement with alternating positive and negative charges, similar to a one-dimensional ionic crystal. These charged residues are hydrated by interior water molecules. The combination of these two effects could explain the stability of such parallel $\beta$-sheet models in spite of the parallel arrangement of charges in amyloid fibrils, which would otherwise suggest destabilization.

Our MD simulations show also that the protofilaments corresponding to the parallel $\beta$-sheet models result in fibrils with a left-handed twist. While fundamentally different from the $\beta$-helical conformations considered by other studies, ${ }^{36,51}$ the twisted conformation of our fibril models also allows for a spatial proximity of the $\mathrm{C}$ and $\mathrm{N}$ termini of adjacent $A \beta_{9-40}$ molecules. Importantly, we find that the protofilament dimeric layers formed by two $A \beta_{9-40}$ molecules do not remain planar. Instead, $\beta$-sheet formation and packing lead to interlocking configurations of staggered $\beta$-strands. It is conceivable that with enhanced staggering, fibril structures essentially without contacts between the two $\beta$-strands of the same $\mathrm{A} \beta$ molecule could form.

Our results suggest a mass-per-length of $\approx 18$ $18.4 \mathrm{kDa} / \mathrm{nm}$. Higher values of $20-22 \mathrm{kDa} / \mathrm{nm}$ would be inconsistent with a $4.8 \AA$ layer spacing in X-ray measurements unless additional mass had adsorbed. On a long scale, we predict a distance of about 50-200 nm per full turn of the twisted fibrils, consistent with electron microscope images of various $A \beta_{1-40}$ and $A \beta_{10-35}$ fibrils. $4,12,27$

Calculated X-ray fiber diffraction patterns for both short protofilament structures from MD trajectory snapshots and for longer, reconstructed fibrils, show the cross- $\beta$ pattern characteristic of packed $\beta$-sheets seen in experiments. They demonstrate that the protofilament structures in our MD simulations and the structural models of long, reconstructed fibrils are in qualitative agreement with experimental X-ray fiber diffraction data. Our calculations also suggest that the equatorial reflections could be sensitive to detailed structural differences between different molecular fibril topologies, such as between the "flipped" (i.e. $F C_{2}^{x}$, $F C_{2}^{z}$ ) and "un-flipped" (i.e. $C_{2}^{x}, C_{2}^{z}$ ) cases. Future $X$-ray fiber diffraction experiments could test this observation.
In summary, our MD simulations show structural stability of $A \beta_{1-40}$ protofilaments formed by dimeric layers of U-shaped peptides with parallel $\beta$-sheets along the fibril axis. Tight packing of the interior side-chains and strong hydrophobic interactions between the $\beta$-strands are critical elements of the resulting structures. In addition, stacked Asp23 and Lys28 residues form a chain of interior salt-bridges, with alternating positive and negative charges reminiscent of a one-dimensional ionic crystal. In simulations of finite protofilaments, we find that these buried charges are partially hydrated through the formation of a narrow interior water channel. Consistent with the structural polymorphism observed in recent experiments, ${ }^{4}$ our simulations show that four different topologies for the interpeptide packing result in stable protofilament structures. The simulation structures appear to be consistent with NMR and X-ray diffraction data. Future experiments may help discriminate between the different fibril topologies and could reveal further details of amyloid fibril structures.

\section{Methods}

\section{Terminology}

To avoid ambiguity, we use the following terminology to discriminate between various types of molecular organization in amyloid aggregates. The term "fibril" is used as a generic term for structures made of aggregated peptides that have a rod-like appearance. A fibril has the lowest degree of organization or its degree of molecular organization is unknown. ${ }^{71}$ A typical feature of amyloid fibrils is their significant $\beta$-sheet content. At a higher degree of organization, two or more fibrils can assemble laterally to form "filaments". The term "protofilament" is used, therefore, to describe the basic structural unit of a structurally ordered amyloid filament or fibril. This is quite different from the term "protofibril" that is used generically for peptide aggregates that are not fully formed fibrils, and might not have a fibril-like appearance. According to this terminology, the structures investigated here are "protofilaments", basic structural units consisting of $A \beta_{9-40}$ peptide monomers arranged in two parallel strands.

\section{Molecular dynamics}

The molecular dynamics simulations of solvated amyloid fibrils were performed using the NAMD2 program. ${ }^{72}$ We used the CHARMM27, ${ }^{73}$ and AMBER94 ${ }^{74}$ force fields, as well as the AMBER94 force field modified by García \& Sanbonmatsu ${ }^{75}$ to better balance the equilibrium between $\alpha$-helical and extended peptide conformers (notation $F C_{2}^{x}$-AGS, Table 1). The fibrils were explicitly solvated with TIP3P water molecules. ${ }^{47,76}$ Simulations were performed in the NPT ensemble.

The Langevin piston $\operatorname{method}^{72,77,78}$ was used to maintain a constant pressure of $1 \mathrm{~atm}(=101,325 \mathrm{~Pa})$. The temperature $(298 \mathrm{~K})$ was controlled by using Langevin dynamics with a coupling coefficient of $1 \mathrm{ps}^{72}$ We used periodic boundary conditions and the particle mesh Ewald (PME) method ${ }^{79}$ with a real-space cutoff 
distance of $10 \AA$ and a grid width of $0.76-0.83 \AA$. The switching distance for non-bonded electrostatics and van der Waals interactions was $8.5 \mathrm{~A}$, and the integration time-step was $1 \mathrm{fs}$.

Initial all-atom conformations were generated based on experimentally derived NMR models. ${ }^{10}$ A backbonedependent rotamer library, SCWRL $3,{ }^{80}$ was used to build initial side-chain configurations. Infinitely long fibrils were simulated by replicating $C_{2}^{x}$ or $C_{2}^{z}$ aligned peptide dimers along the $z$ axis with a $4.8 \AA$ spacing between different layers, as suggested by the experimental model, in either the original or "flipped" configurations (i.e. $F C_{2}^{x}$ and $\left.F C_{2}^{z}\right)$. As shown in Figure 1, the flipped configurations were generated by rotating the backbone $\Phi$ and $\Psi$ dihedral angles in the vicinity of the G29 and A30 residues, such that the residues responsible for intermolecular side-chain interactions in the dimeric layers are I31-G33-M35-G37-V39 instead of I32-L34-V36-G38-V40.

We simulated both finite fibril fragments of $4 \times 2$ peptides (Figure 2(a)) and infinitely long fibrils aligned with the $z$ axis (Figure 2(b)). In the simulations of infinitely long fibrils, the periodic boundary conditions do not allow the fibrils to twist.

The initial structures were first energy minimized, then the fibrils were hydrated. The resulting systems were gradually heated to a temperature of $298 \mathrm{~K}$ at a pressure of $1 \mathrm{~atm}$, for about $10 \mathrm{ps}$. Finally, NPT MD runs were used to investigate the structural dynamics and conformational stability on a time-scale of $\approx 10 \mathrm{~ns}$ for systems ranging from 17,000 atoms to over 52,000 atoms (see Table 1). The data visualization was carried out using $\mathrm{VMD}^{81}$ and Raster3D. ${ }^{82}$

\section{Fibril topology and symmetry}

In our finite fibril simulations, we studied systems of four layers along the fibril axis. We used the structure of the central two layers to estimate the topology of a long fibril by constructing a rigid-body symmetry transformation connecting one layer to the next along the fibril axis. Recursive application of this transformation then allowed us to build a long fibril that has the same two-layer steps as the short, simulated fibril.

We use the term "rigid-body" for a transformation consisting only of finite rotations and translations that leaves the peptide conformation unchanged. $\mathbf{x}_{0}$ denotes a coordinate vector of an atom in the amyloid fibril dimeric layer (i.e. two $A \beta$ peptides). The corresponding coordinate vector $\mathbf{x}_{1}$ in the second fibril dimeric layer is obtained by applying a rotation and a translation:

$$
\mathbf{x}_{1}=\mathbf{R} \mathbf{x}_{0}+\mathbf{t}
$$

where $\mathbf{R}$ is a rotation matrix and $\mathbf{t}$ is a translation vector. Figure 6(a) shows a schematic representation of this rigidbody transformation. The atomic coordinates $x_{1}$ in the second layer of the fiber are thus obtained by first rotating the coordinates of the previous layer $x_{0}$ around a rotation axis $\phi$ by an angle $\phi=|\phi|$, and then by translating the rotated coordinates by $t$. Recursive applications of this transformation gives the atomic coordinates of the $n$th layer as:

$$
\mathbf{x}_{n}=\mathbf{R}^{n} \mathbf{x}_{0}+\sum_{i=0}^{n-1} \mathbf{R}^{i} \mathbf{t}=\mathbf{R}^{n} \mathbf{x}_{0}+\mathbf{t}_{n}
$$

In a more compact form, we have $\mathbf{t}_{n}=(\mathbf{R}-\mathbf{I})^{-1}\left(\mathbf{R}^{n}-\mathbf{I}\right) \mathbf{t}$, where $\mathbf{I}$ is the 3D identity matrix.

To extract the rigid-body symmetry transformation $(\mathbf{R}, \mathbf{t})$ from the MD simulation trajectories, we repeatedly analyzed the layer structure of the amyloid fibrils. For a given fibril structure, we determined the transformation $(\mathbf{R}, \mathbf{t})$ from an RMSD alignment of peptide monomers in the third layer with respect to that in the second layer. The translation vector is simply the distance vector between the centers of geometry of the two layers. The optimal rotation matrix $\mathbf{R}$ that minimizes the RMSD is computed by using a singular value decomposition method. ${ }^{33}$ The rotation angle is given by $\cos |\phi|=(\operatorname{Tr}(\mathbf{R})-1) / 2$ The corresponding rotation axis is the eigenvector of $\mathbf{R}$ for eigenvalue $\lambda=1,{ }^{84}$ where we chose $|\phi|$ to be the angle of the rotation. For the RMSD alignment we used the $\alpha$ carbon atoms of core residues (e.g. 15-22 and 31-38 in the $\beta$-strand region, or 22-29 for the loop). To provide a reference frame, configurations were aligned to the initial structure, such that the $z$-axis is roughly normal to the first peptide dimer plane.

\section{Mass-per-length estimates for twisted fibrils}

Fibrils obtained by recursive application of the rigidbody transformation in equation (2) are oriented along the direction of the rotation axis $\phi$. As shown by the data extracted from all-atom MD simulations, this direction can deviate from the translation vector $\mathbf{t}$ connecting the geometric centers of adjacent peptide layers. The massper-length values of the fibrils are thus, in general, larger than for fibrils in which the fibril axis and the translation vectors coincide.

Along the fibril axis, the step height between two dimeric layers is given by:

$$
h=\frac{|\mathbf{t} \phi|}{|\phi|}
$$

Therefore, if $M$ is the mass of a single fibril layer (here: peptide dimer), the mass-per-length (MPL) of a twisted fibril is given by:

$$
\text { MPL }=\frac{M}{h}
$$

MPL values corresponding to long fibrils reconstructed based on parameters extracted from our MD simulations are given in Table 2 .

Note that if the translation and rotation axes have the same direction (i.e. if $\mathbf{t} /|\mathbf{t}|= \pm \phi /|\phi|)$, then $h=|\mathbf{t}|$, and $\mathrm{MPL}=M /|\mathbf{t}|$ as expected. We can also estimate the "pitch" $P$ (i.e. the distance along the fibril axis for a full helical turn) as $P=2 \pi h /|\phi|$.

\section{Calculated X-ray fiber diffraction pattern}

The X-ray fiber diffraction patterns in Figure 8(a) were computed using the Diffraction-Amorphous module of the Cerius ${ }^{2}$ software $\dagger$. With this method, we calculated the $2 \mathrm{D}$ cylindrically averaged scattering intensity. The fibrils were aligned with their axes along the $z$ direction.

The calculated scattering pattern is a representation in polar coordinates $(Q, \alpha)$ of the scattering intensity $I(Q, \alpha)$, where $Q=4 \pi \sin (\theta) / \lambda$ is the scattering vector, $2 \theta$ is the scattering angle, $\lambda$ is the radiation wavelength, and $\alpha$ is the angle measured from the meridian. We used the default value for the $\mathrm{X}$-ray beam wavelength $\lambda=1.54178 \AA(\mathrm{Cu} \mathrm{K})$.

The pattern in Figure $8(\mathrm{a})$ was calculated using the

$\dagger$ Accelrys Inc. Cerius2, version 4.8.

http:/ /www.accelrys.com/ 
Legendre scattering method. The scattering intensity was approximated by a sum over the first five terms of a Legendre series expansion:

$$
I(Q, \alpha)=\sum_{n=0}^{4} w_{2 n} I_{2 n}(Q) P_{2 n}(\cos \alpha)
$$

where higher-order weights are always zero. We used the default values of the Legendre weights $\left(w_{0}=1.00\right.$, $w_{2}=0.75, w_{4}=0.50, w_{6}=0.25, w_{8}=0.00$ ). Figure $8(\mathrm{a})$ shows a " $Q$-weighted reduced scattering" representation of the diffraction patterns $(Q i(Q))$, where the "reduced scattering" $(i(Q))$ represents the total scattering $(I(Q))$ minus the independent coherent scattering component $\dagger$.

Our calculations involved the following steps: (1) for each of the four finite segment trajectories (i.e. $C_{2}^{x}, F C_{2}^{x}, C_{2}^{z}$ and $\left.F C_{2}^{z}\right)$, instantaneous structures were extracted and aligned such that their fibril axes (extracted from the central peptide layers) were aligned to the $z$-axis. The structures were extracted from the $5 \mathrm{~ns}$ to $10 \mathrm{~ns}$ parts of the trajectories, every $0.2 \mathrm{~ns}$; (2) a " $Q$-weighted reduced scattering pattern", $Q i(Q)$, was calculated using the Cerius $^{2}$ software $\dagger$ as described above, for each of the aligned instantaneous structures extracted from each trajectory; (3) finally, an average $Q$-weighted reduced scattering pattern $\langle Q i(Q)\rangle$ was calculated for the 26 instantaneous scattering patterns obtained for each fibril topology (as shown in Figure 8(a)).

\section{Acknowledgements}

N.V.B. \& G.H. thank Dr Christine Peter-Tittelbach and Dr In-Chul Yeh for many helpful discussions. This research was supported by the Intramural Research Program of the National Institute of Diabetes and Digestive and Kidney Diseases, National Institutes of Health.

\section{Supplementary Data}

Supplementary data associated with this article can be found, in the on-line version, at 10.1016/j. jmb.2005.08.066

\section{References}

1. Sunde, M. \& Blake, C. C. F. (1998). From the globular to the fibrous state: protein structure and structural conversion in amyloid formation. Quart. Rev. Biophys. 31, 1-39.

2. Caughey, B. \& Lansbury, P. T. (2003). Protofibrils, pores, fibrils, and neurodegeneration: separating the responsible protein aggregates from the innocent bystanders. Annu. Rev. Neurosci. 26, 267-298.

3. Ahmad, A., Millett, I. S., Doniach, S., Uversky, V. N. \& Fink, A. L. (2004). Stimulation of insulin fibrillation by urea-induced intermediates. J. Biol. Chem. 279, 14999-15013.

4. Petkova, A. T., Leapman, R. D., Guo, Z. H., Yau, W. M., Mattson, M. P. \& Tycko, R. (2005). Selfpropagating, molecular-level polymorphism in Alzheimer's $\beta$-amyloid fibrils. Science, 307, 262-265.
5. Tycko, R. (2004). Progress towards a molecular-level structural understanding of amyloid fibrils. Curr. Opin. Struct. Biol. 14, 96-103.

6. Wetzel, R. (2002). Structure. Biochemistry, 10, 1031-1036.

7. Scheibel, T., Parthasarathy, R., Sawicki, G., Lin, X.-M., Jaeger, H. \& Lindquist, S. L. (2003). Conducting nanowires built by controlled self-assembly of amyloid fibers and selective metal deposition. Proc. Natl Acad. Sci. USA, 100, 4527-4532.

8. MacPhee, C. E. \& Dobson, C. M. (2000). Formation of mixed fibrils demonstrates the generic nature and potential utility of amyloid nanostructures. J. Am. Chem. Soc. 122, 12707-12713.

9. Reches, M. \& Gazit, E. (2003). Casting metal nanowires within discrete self-assembled peptide nanotubes. Science, 300, 625-627.

10. Petkova, A. T., Ishii, Y., Balbach, J. J., Antzutkin, O. N., Leapman, R. D., Delaglio, F. \& Tycko, R. (2002). A structural model for Alzheimer's beta-amyloid fibrils based on experimental constraints from solid state NMR. Proc. Natl Acad. Sci. USA, 99, 16742-16747.

11. Tycko, R. (2003). Insights into the amyloid folding problem from solid-state NMR. Biochemistry, 42, 3151-3159.

12. Antzutkin, O. N., Leapman, R. D., Balbach, J. J. \& Tycko, R. (2002). Supramolecular structural constraints on Alzheimer's beta-amyloid fibrils from electron microscopy and solid-state nuclear magnetic resonance. Biochemistry, 41, 15436-15450.

13. Antzutkin, O. N., Balbach, J. J., Leapman, R. D., Rizzo, N. W., Reed, J. \& Tycko, R. (2000). Multiple quantum solid-state NMR indicates a parallel, not antiparallel, organization of $\beta$-sheets in Alzheimer's $\beta$-amyloid fibrils. Proc. Natl Acad. Sci. USA, 97, 13045-13050.

14. Balbach, J. J. A. T., Petkova, N. A. O., Antzutkin, O. N., Gordon, D. J., Meredith, S. C. \& Tycko, R. (2002). Supramolecular structure in full-length Alzheimer's beta-amyloid fibrils: evidence for a parallel beta-sheet organization from solid-state nuclear magnetic resonance. Biophys. J. 83, 1205-1216.

15. Benzinger, T. L., Gregory, D. M., Burkoth, T. S., MillerAuer, H., Lynn, D. G., Botto, R. E. \& Meredith, S. C. (1998). Propagating structure of Alzheimer's $\beta$-amyloid (10-35) is parallel $\beta$-sheet with residues in exact register. Proc. Natl Acad. Sci. 95, 13407-13412.

16. Gregory, D. M., Benzinger, T. L. S., Burkoth, T. S., Miller-Auer, H., Lynn, D. G., Meredith, S. C. \& Botto, R. E. (1998). Dipolar recoupling NMR of biomolecular self-assemblies: determining inter- and intrastrand distances in fibrilized Alzheimer's beta-amyloid peptide. Solid State Nucl. Magn. Reson. 13, 149-166.

17. Lansbury, P. T., Costa, P. R., Griffiths, J. M., Simon, E. J., Auger, M., Halverson, K. J. et al. (1995). Structural model for the beta-amyloid fibril based on interstrand alignment of an antiparallel-sheet comprising a C-terminal peptide. Nature Struct. Biol. 2, 990-998.

18. Balbach, J. J., Ishii, Y., Antzutkin, O. N., Leapman, R. D., Rizzo, N. W., Dyda, F. et al. (2000). Amyloid fibril formation by $A \beta$ (16-22), a seven-residue fragment of the Alzheimer's $\beta$-amyloid peptide, and structural characterization by solid state NMR. Biochemistry, 39, 13748-13759.

19. Petkova, A. T., Buntkowsky, G., Dyda, F., Leapman, R. D., Yau, W. M. \& Tycko, R. (2004). Solid state NMR reveals a pH-dependent antiparallel beta-sheet registry in fibrils formed by a beta-amyloid peptide. J. Mol. Biol. 335, 247-260. 
20. Tycko, R. \& Ishii, Y. (2003). Constraints on supramolecular structure in amyloid fibrils from twodimensional solid-state NMR spectroscopy with uniform isotopic labeling. J. Am. Chem. Soc. 125, 6606-6607.

21. Antzutkin, O. N., Balbach, J. J. \& Tycko, R. (2003). Sitespecific identification of non- $\beta$-strand conformations in Alzheimer's $\beta$-amyloid fibrils by solid-state NMR. Biophys. J. 84, 3326-3335.

22. Torok, M., Milton, S., Kayed, R., Wu, P., McIntire, T., Glabe, C. G. \& Langen, R. (2002). Structural and dynamic features of Alzheimer's $A \beta$ peptide in amyloid fibrils studied by site-directed spin labeling. J. Biol. Chem. 277, 40810-40815.

23. Khurana, R., Ionescu-Zanetti, C., Pope, M., Li, J., Nielson, L., Ramirez-Alvarado, M. et al. (2003). A general model for amyloid fibril assembly based on morphological studies using atomic force microscopy. Biophys. J. 85, 1135-1144.

24. Kheterpal, I., Lashuel, H. A., Hartley, D. M., Walz, T., Lansbury, P. T., Jr \& Wetzel, R. (2003). A $\beta$ protofibrils possess a stable core structure resistant to hydrogen exchange. Biochemistry, 42, 14092-14098.

25. Williams, A. D., Portelius, E., Kheterpal, I., Guo, J.-T., Cook, K. D., Xu, Y. \& Wetzel, R. (2004). Mapping A $\beta$ amyloid fibril secondary structure using scanning proline mutagenesis. J. Mol. Biol. 335, 833-842.

26. Fernandez-Escamilla, A.-M., Rousseau, F., Schymkowitz, J. \& Serrano, L. (2004). Prediction of sequence-dependent and mutational effects on the aggregation of peptides and proteins. Nature Biotechnol. 22, 1302-1306.

27. Goldsbury, C. S., Wirtz, S., Muller, S. A., Sunderji, S., Wicki, P., Aebi, U. \& Frey, P. (2000). Studies on the in vitro assembly of $A \beta$ 1-40: implications for the search for A $\beta$ fibril formation inhibitors. J. Struct. Biol. 130, 217-231.

28. Massi, F., Peng, J. W., Lee, J. P. \& Straub, J. E. (2001). Simulation study of the structure and dynamics of the Alzheimer's amyloid peptide congener in solution. Biophys. J. 80, 31-44.

29. Straub, J. E., Guevara, J., Huo, S. H. \& Lee, J. P. (2002). Long time dynamic simulations: exploring the folding pathways of an Alzheimer's amyloid A $\beta$-peptide. Accts Chem. Res. 35, 473-481.

30. Ma, B. Y. \& Nussinov, R. (2002). Stabilities and conformations of Alzheimer's $\beta$-amyloid peptide oligomers $(A \beta$ (16-22), $A \beta(16-35)$ and $A \beta(10-35))$ : sequence effects. Proc. Natl Acad. Sci. USA, 99, 14126-14131.

31. Klimov, D. K. \& Thirumalai, D. (2003). Dissecting the assembly of $A \beta$ (16-22) amyloid peptides into antiparallel $\beta$ sheets. Structure, 11, 295-307.

32. Hwang, W., Zhang, S., Kamm, R. D. \& Karplus, M. (2004). Kinetic control of dimer structure formation in amyloid fibrillogenesis. Proc. Natl Acad. Sci. USA, 101, 12916-12921.

33. Klimov, D. K., Straub, J. E. \& Thirumalai, D. (2004). Aqueous urea solution destabilizes $\mathrm{A} \beta_{16-22}$ oligomers. Proc. Natl Acad. Sci. USA, 101, 14760-14765.

34. Urbanc, B., Cruz, L., Ding, F., Sammond, D., Khare, S., Buldyrev, S. V. et al. (2004). Molecular dynamics simulation of amyloid $\beta$ dimer formation. Biophys. J. 87, 2310-2321.

35. Tarus, B., Straub, J. E. \& Thirumalai, D. (2005). Probing the initial stage of aggregation of the $\mathrm{A} \beta_{10-35}$-protein: assessing the propensity for peptide dimerization. J. Mol. Biol. 345, 1141-1156.
36. Guo, J.-T., Wetzel, R. \& Xu, Y. (2004). Molecular modeling of the core of $A \beta$ amyloid fibrils. Proteins: Struct. Funct. Genet. 57, 357-364.

37. Xu, Y., Shen, J., Luo, X., Zhu, W., Chen, K., Ma, J. \& Jiang, H. (2005). Conformational transition of amyloid ß-peptide. Proc. Natl Acad. Sci. USA, 102, 5403-5407.

38. Lazo, N. D. \& Downing, D. T. (1998). Amyloid fibrils may be assembled from beta-helical protofibrils. Biochemistry, 3, 1731-1735.

39. Mager, P. P. (1998). Molecular simulation of the primary and secondary structures of the $A \beta(1-42)$ peptide of Alzheimer's disease. Med. Res. Rev. 18, 403-430.

40. Tjernberg, L. O., Callaway, D. J. E., Tjernberg, A., Hahne, S., Lilliehook, C., Terenius, L. et al. (1999). A molecular model of Alzheimer amyloid betapeptide fibril formation. J. Biol. Chem. 274, 12619-12625.

41. Tjernberg, L. O., Tjernberg, A., Bark, N., Shi, Y., Ruzsicska, B. P., Bu, Z. M. et al. (2002). Assembling amyloid fibrils from designed structures containing a significant amyloid beta-peptide fragment. Biochem. J. 366, 343-351.

42. Li, L., Darden, T. A., Bartolotti, L., Kominos, D. \& Pedersen, L. G. (1999). An atomic model for the pleated $\beta$-sheet structure of A $\beta$ amyloid protofilaments. Biophys. J. 76, 2871-2878.

43. George, A. R. \& Howlett, D. R. (1999). Computationally derived structural models of the beta-amyloid found in Alzheimer's disease plaques and the interaction with possible aggregation inhibitors. Biopolymers, 50, 733-741.

44. Chaney, M. O., Webster, S. D., Kuo, Y. M. \& Roher, A. E. (1998). Molecular modeling of the $A \beta_{1-42}$ peptide from Alzheimer's disease. Protein Eng. 11, 761-767.

45. Kheterpal, I., Williams, A., Murphy, C., Bledsoe, B. \& Wetzel, R. (2001). Structural features of the A $\beta$ amyloid fibril elucidated by limited proteolysis. Biochemistry, 40, 11757-11767.

46. Kheterpal, I., Wetzel, R. \& Cook, K. D. (2003). Enhanced correction methods for hydrogen exchange-mass spectrometric studies of amyloid fibrils. Protein Sci. 12, 635-643.

47. Mahoney, M. W. \& Jorgensen, W. L. (2000). A five-site model for liquid water and the reproduction of the density anomaly by rigid, nonpolarizable potential functions. J. Chem. Phys. 112, 8910-8922.

48. Whittemore, N. A., Mishra, R., Kheterpal, I., Williams, A. D., Wetzel, R. \& Serpersu, E. H. (2005). Hydrogendeuterium (H/D) exchange mapping of $A \beta_{1-40}$ amyloid fibril secondary structure using nuclear magnetic resonance spectroscopy. Biochemistry, 44, 4434-4441.

49. Cornilescu, G., Delaglio, F. \& Bax, A. (1999). Protein backbone angle restraints from searching a database for chemical shift and sequence homology. J. Biomol. NMR, 13, 289-302.

50. Malinchik, S. B., Inouye, H., Szumowski, K. E. \& Kirschner, D. A. (1998). Structural analysis of Alzheimer's $\beta(1-40)$ amyloid: protofilament assembly of tubular fibrils. Biophys. J. 74, 537-545.

51. Perutz, M. F., Finch, J. T., Berriman, J. \& Lesk, A. (2002). Amyloid fibers are water-filled nanotubes. Proc. Natl Acad. Sci. USA, 99, 5591-5595.

52. Kishimoto, A., Hasegawa, K., Suzuki, H., Taguchi, H., Namba, K. \& Yoshida, M. (2004). $\beta$-Helix is a likely core structure of yeast prion Sup35 amyloid fiber. Biochem. Biophys. Res. Commun. 315, 739-745. 
53. Tsodikov, O. V., Record, M. T. \& Sergeev, Y. V. (2002). Novel computer program for fast exact calculation of accessible and molecular surface areas and average surface curvature. J. Comput. Chem. 23, 600-609.

54. Lins, L., Thomas, A. \& Brasseur, R. (2003). Analysis of accessible surface of residues in proteins. Protein Sci. 12, 1406-1417.

55. Berman, H. M., Westbrook, J., Feng, Z., Gilliland, G., Bhat, T. N., Weissig, H. et al. (2000). The Protein Data Bank. Nucl. Acids Res. 28, 235-242.

56. Liwo, A., Oldziej, S., Pincus, M. R., Wawak, R. J., Rackovsky, S. \& Scheraga, H. A. (1997). A unitedresidue force field for off-lattice protein-structure simulations. I. Functional forms and parameters of long-range side-chain interaction potentials from protein crystal data. J. Comput. Chem. 18, 849-873.

57. Buchete, N.-V., Straub, J. E. \& Thirumalai, D. (2004). Orientational potentials extracted from protein structures improve native fold recognition. Protein Sci. 13, 862-874.

58. Richards, F. M. (1977). Areas, volumes, packing, and protein-structure. Annu. Rev. Biophys. Biol. 6, 151-176.

59. Samanta, U., Bahadur, R. P. \& Chakrabarti, P. (2002). Quantifying the accessible surface area of protein residues in their local environment. Protein Eng. 15, 659-667.

60. García, A. E. \& Hummer, G. (2000). Water penetration and escape in proteins. Proteins: Struct. Funct. Genet. 38, 261-272.

61. Halle, B. \& Davidovic, M. (2003). Biomolecular hydration: from water dynamics to hydrodynamics. Proc. Natl Acad. Sci. USA, 100, 12135-12140.

62. Lakdawala, A. S., Morgan, D. M., Liotta, D. C., Lynn, D. G. \& Snyder, J. P. (2002). Dynamics and fluidity of amyloid fibrils: a model of fibrous protein aggregates. J. Am. Chem. Soc. 124, 15150-15151.

63. Hummer, G., Rasaiah, J. C. \& Noworyta, J. P. (2001). Water conduction through the hydrophobic channel of a carbon nanotube. Nature, 414, 188-190.

64. Thirumalai, D., Klimov, D. K. \& Dima, R. I. (2003). Emerging ideas on the molecular basis of protein and peptide aggregation. Curr. Opin. Struct. Biol. 13, 146-159.

65. Dima, R. I. \& Thirumalai, D. (2004). Proteins associated with diseases show enhanced sequence correlation between charged residues. Bioinformatics, 20, 2345-2354.

66. Otzen, D. E., Kristensen, O. \& Oliveberg, M. (2000). Designed protein tetramer zipped together with a hydrophobic Alzheimer homology: a structural clue to amyloid assembly. Proc. Natl Acad. Sci. USA, 97, 9907-9912.

67. Richardson, J. S. \& Richardson, D. C. (2002). Natural $\beta$-sheet proteins use negative design to avoid edge-toedge aggregation. Proc. Natl Acad. Sci. USA, 99, 2754-2759.

68. Eaton, W. A. \& Hofrichter, J. (1995). The biophysics of sickle-cell hydroxyurea therapy. Science, 268, 1142-1143.
69. Christoph, G. W., Hofrichter, J. \& Eaton, W. A. (2005). Understanding the shape of sickled red cells. Biophys. J. 88, 1371-1376.

70. Perutz, M. F., Pope, B. J., Owen, D., Wanker, E. E. \& Scherzinger, E. (2002). Aggregation of proteins with expanded glutamine and alanine repeats of the glutamine-rich and asparagine-rich domains of Sup35 and of the amyloid beta-peptide of amyloid plaques. Proc. Natl Acad. Sci. USA, 99, 5596-5600.

71. Zanuy, D., Gunasekaran, K., Ma, B. Y., Tsai, H. H., Tsai, C. J. \& Nussinov, R. (2004). Insights into amyloid structural formation and assembly through computational approaches. Amyloid: J. Protein Fold. Disord. 11, 143-161.

72. Kale, L., Skeel, R., Bhandarkar, M., Brunner, R., A, G., Krawetz, N., Phillips, J. et al. (1999). NAMD2: greater scalability for parallel molecular dynamics. J. Comput. Phys. 151, 283-312.

73. MacKerell, A. D., Bashford, D., Bellott, M., Dunbrack, R. L., Evanseck, J. D., Field, M. J. et al. (1998). All-atom empirical potential for molecular modeling and dynamics studies of proteins. J. Phys. Chem. B, 102, 3586-3616.

74. Cornell, W. D., Cieplak, P., Bayly, C. I., Gould, I. R., Merz, K. M., Ferguson, D. M. et al. (1995). A 2nd generation force-field for the simulation of proteins, nucleic-acids, and organic-molecules. J. Am. Chem. Soc. 117, 5179-5197.

75. García, A. E. \& Sanbonmatsu, K. Y. (2002). Alphahelical stabilization by side chain shielding of backbone hydrogen bonds. Proc. Natl Acad. Sci. USA, 99, 2782-2787.

76. Jorgensen, W. L., Chandrasekhar, J., Madura, J. D., Impey, R. W. \& Klein, M. L. (1983). Comparison of simple potential functions for simulating liquid water. J. Chem. Phys. 79, 926-935.

77. Martyna, G. J., Tobias, D. J. \& Klein, M. L. (1994). Constant-pressure molecular-dynamics algorithms. J. Chem. Phys. 101, 4177-4189.

78. Feller, S. E., Zhang, Y. H., Pastor, R. W. \& Brooks, B. R. (1995). Constant-pressure molecular-dynamics simulation-the Langevin piston method. J. Chem. Phys. 103, 4613-4621.

79. Darden, T., York, D. \& Pedersen, L. (1993). Particle Mesh Ewald-an $N \log (N)$ method for Ewald sums in large systems. J. Chem. Phys. 98, 10089-10092.

80. Canutescu, A. A., Shelenkov, A. A. \& Dunbrack, R. L. (2003). A graph-theory algorithm for rapid protein side-chain prediction. Protein Sci. 12, 2001-2014.

81. Humphrey, W., Dalke, A. \& Schulten, K. (1996). VMD-visual molecular dynamics. J. Mol. Graph. 14, 33-38.

82. Merritt, E. A. \& Bacon, D. J. (1997). Raster3D: photorealistic molecular graphics. Methods Enzymol. $277,505-524$.

83. Golub, G. H. \& Loan, C. F. V. (1996). Matrix Computations, Johns Hopkins University Press, Baltimore, MD.

84. Goldstein, H. (1980). Classical Mechanics, AddisonWesley, Reading, MA. 